\title{
Four new species of Heteromysis (Crustacea: Mysida) from public aquaria in Hawaii, Florida, and Western to Central Europe
}

\author{
Karl J. WITTMANN ${ }^{1, *}$ \& Daniel ABED-NAVANDI ${ }^{2}$ \\ ${ }^{1}$ Medical University of Vienna, Department of Environmental Health, Kinderspitalgasse 15, \\ 1090 Vienna, Austria. \\ ${ }^{2}$ Haus des Meeres - Aqua Terra Zoo, Fritz Grünbaum Platz 1, 1060 Vienna, Austria. \\ *Corresponding author: karl.wittmann@meduniwien.ac.at \\ 2Email: daniel.abed@haus-des-meeres.at \\ ${ }^{1}$ urn:1sid:zoobank.org:author:C90E7BC4-A27A-4B41-93F3-6224D17795FF \\ ${ }^{2}$ urn:lsid:zoobank.org:author:179B83B0-8C8B-4DF5-8986-4227B8E1BB9B
}

\begin{abstract}
Four new species of the subgenus Heteromysis (Olivemysis) were detected in material from (sub)-tropical aquaria in six public aquarium institutions around the globe. Modifications of pleopods by spines represent the strongest structural complex used for differentiation within this subgenus: male pleopods 1-4 modified in $H$. smithsoniana sp. nov., male pleopods 2-4 plus female pleopod 2 in $H$. hornimani sp. nov. and $H$. waikikensis sp. nov. Additional important diagnostic characters are provided by the antennulae, uropods, and telson. The male of $H$. sixi sp. nov. represents a very rare case within the genus Heteromysis by having only pleopod 2 modified by flagellate spines. The definition of the subgenus Olivemysis is modified in order to include H. sixi sp. nov. A summary of pleopod modifications in the genus Heteromysis and a key to the species of the subgenus Olivemysis are given. The here described new taxa more than double the number of Heteromysis species known from aquaria yet unknown in nature from three to seven.
\end{abstract}

Keywords. First description, taxonomy, public aquarium institutions, modified pleopods, key to species.

Wittmann K.J. \& Abed-Navandi D. 2021. Four new species of Heteromysis (Crustacea: Mysida) from public aquaria in Hawaii, Florida, and Western to Central Europe. European Journal of Taxonomy 735: 133-175. https://doi.org/10.5852/ejt.2021.735.1247

\section{Introduction}

The first records of species of the mysid genus Heteromysis S.I. Smith, 1873, in public aquaria were reported by Schlacher et al. (1992) and Ariani et al. (1993) in the frame of biomineralogical studies on statoliths in species of Mysidae Haworth, 1825, including material of Heteromysis mayana Brattegard, 1970, from the coral reef lagoon aquarium at the former (1980-1998) "Exploring Marine Ecosystems Exhibit" at the Smithsonian National Museum of Natural History, Washington, DC. One decade later, Murano \& Fukuoka (2003) presented first descriptions of H. (Olivemysis) kushimotensis and H. (Heteromysis) nomurai from the Kushimoto Marine Park Center, Japan, the latter also found in 
nature. This was followed by descriptions of $H$. (O.) domusmaris Wittmann \& Abed-Navandi, 2019, and $H$. (H.) abednavandii Wittmann, 2020, from the Haus des Meeres Aquarium in Vienna, Austria. At this point, the surprise solidified that as many as three new species of this genus were reported only from aquarium systems, although 90 species are known from nature (census from 18 Oct. 2020). However, a potential selection bias in the relative frequencies of aquarium versus nature taxa cannot be excluded because the potential occurrence of field species in aquaria may have been recognized but not yet published. To shed light on the enigma of 'missing' field species, we initiated a project collecting and analysing material from a great number of aquarium institutions, some of which acquire their material from remote sea areas via exchange partners, collectors, or wholesalers. So far, we have obtained known field species of a few other genera but not of Heteromysis. Here, we present the first descriptions of four additional species of Heteromysis so far not found in nature. The data stock is still growing, and complete sampling lists, additional species descriptions, and analyses will be published elsewhere.

\section{Material and methods}

\section{Material and data request}

In 2019 and 2020 a total of 2023 aquarium institutions was contacted using the listserver "Aquaticinfo. Thelist - An international forum of professional aquarists". The members of the mailing list were asked for sightings and potential samples to be included in the present project. This non-personalized query yielded a reply rate of less than $1 \%$. The survey was continued with a personalized mailing survey by contacting public institutions, private aquarium keepers, and marine wholesalers. Overall, positive replies were so far received from 31 institutions. Up to the present contribution, a total of 52 samples was received, and we expect additional ones. Most responders were previously unaware of these animals, which presumably invaded the aquaria as hitch-hikers in hollows of damp or submerged aquarium substrates upon transport. The responders were also asked for data (Table 1) that could be helpful in tracing the natural origin of the animals.

\section{Sampling}

Sampling was performed in (sub)-tropical aquarium systems by instructed local personnel. The mysids were taken from fish-free service tanks connected with the main aquarium system and from shaded crevices and recesses in the aquarium displays. Most specimens were caught with suction pipes and transferred to ethanol 95\%. A moderate water current which supplied the mysids with floating food particles prevailed in all sampling locations. The video clip in Suppl. file 1, recorded by D.A.N., shows a typical fish-free habitat of a population of Heteromysis abednavandii in the Haus des Meeres Aquarium in Vienna, Austria. Here, accompanying crustaceans are the snapping shrimp Alpheus bellulus Miya \& Miyake, 1969, and a swarm of the copepod Euaugaptilus sp. (det. S. Gaviria). Note that the movements of the crustaceans may have been accelerated by the elevated light intensity during that video shot.

\section{Material studied}

A selection of eight samples from public aquaria is given in Table 1. The remaining samples are subject to ongoing research and will be published elsewhere. Holotypes and slides of dissected paratypes were deposited at the Natural History Museum of Vienna, vial paratypes at this same institution and at the Smithsonian National Museum of Natural History (Washington).

\section{Laboratory methods}

Preparation, measurements, and examination of materials were carried out as described in Wittmann (2000), examination of statolith structure as detailed in Wittmann et al. (1993). The spines on the lateral margins of the telson were counted without terminal spines. Due to the different insertion of 
WITTMANN K.J. \& ABED-NAVANDI D., New species of Heteromysis from public aquaria

Table 1. Samples and data of Heteromysis S.I. Smith, 1873 obtained from aquarium institutions.

\begin{tabular}{|c|c|c|c|c|c|c|}
\hline $\begin{array}{l}\text { Sample } \\
\text { no. }\end{array}$ & Institution & Locality & Tracing & leg. / don. & Date & Species recorded \\
\hline 1 & $\begin{array}{l}\text { Waikiki } \\
\text { Aquarium }\end{array}$ & $\begin{array}{l}\text { Honolulu, } \\
\text { Hawaii, USA }\end{array}$ & $\begin{array}{l}\text { Local input }{ }^{*} \text { from } \\
\text { Central Pacific Ocean }\end{array}$ & Gwen Lentes & Jan. 2020 & $\begin{array}{l}\text { H. waikikensis sp. nov. } \\
\text { H. sixi sp. nov. }\end{array}$ \\
\hline 2 & $\begin{array}{l}\text { Smithsonian } \\
\text { Marine } \\
\text { Ecosystems } \\
\text { Aquarium: } \\
\text { Atlantic coral } \\
\text { reef Exhibit }\end{array}$ & $\begin{array}{l}\text { Fort Pierce, } \\
\text { Florida, USA }\end{array}$ & $\begin{array}{l}\text { Local input from } \\
\text { subtropical NW- } \\
\text { Atlantic }\end{array}$ & Bill Hoffman & Dec. 2019 & H. smithsoniana sp. nov. \\
\hline 3 & $\begin{array}{l}\text { Horniman } \\
\text { Museum } \\
\text { Aquarium }\end{array}$ & London, UK & $\begin{array}{l}\text { Corals acquired from } \\
\text { Tropical Marine } \\
\text { Centre Inc., UK }\end{array}$ & Jamie Craggs & Dec. 2019 & H. hornimani sp. nov. \\
\hline 4 & $\begin{array}{l}\text { Aquarium } \\
\text { de Paris - } \\
\text { Cineaqua }\end{array}$ & Paris, F & $\begin{array}{l}\text { Import of Caribbean } \\
\text { rock (corals) }\end{array}$ & Anaïs Courtet & Sep. 2020 & H. hornimani sp. nov. \\
\hline $5-6$ & $\begin{array}{l}\text { Oceanopolis } \\
\text { Aquarium }\end{array}$ & Brest, F & $\begin{array}{l}\text { Local input from } \\
\text { temperate } \\
\text { NE Atlantic. Import } \\
\text { of Indonesian and } \\
\text { Caribbean corals. }\end{array}$ & $\begin{array}{l}\text { Dominique } \\
\text { Barthelmy }\end{array}$ & Feb. 2020 & H. hornimani sp. nov. \\
\hline$\overline{7-8}$ & $\begin{array}{l}\text { Wroclaw } \\
\text { Zoo }\end{array}$ & Wroclaw, PL & $\begin{array}{l}\text { Via corals from } \\
\text { Burgers Zoo Arnhem } \\
\text { (NL) and Zoo } \\
\text { Hagenbeck (D) }\end{array}$ & Jakub Kordas & $\begin{array}{l}\text { Jul.-Aug. } \\
2020\end{array}$ & H. hornimani sp. nov. \\
\hline
\end{tabular}

* 'Local input' indicates regular input of water, stones and sand from coastal waters near aquarium institution.

morphological structures, their relative extension may differ from relative size, e.g., in Fig. $1 \mathrm{E}$ the exopod is $22 \%$ longer (in terms of exopod length) than the endopod, but extends only $17 \%$ beyond the endopod.

\section{Morphological comparison}

Among the previously published 93 species (census 18 Oct. 2020) plus one non-nominotypical subspecies of the genus Heteromysis considered valid by the present authors, there are 22 taxa with either males or females still undescribed; in three other taxa, only non-adults have been described in any sex. Species definitions and descriptions are elaborated in great detail here in order to cope with the great heterogeneity of published species descriptions in this genus. Photos and drawings of sex-specific features are labelled by symbols for females or males, respectively, provided that both sexes are known. Accordingly, the absence of such labels implies absent or indistinct sex-specific differences.

\section{Terminology}

Terminology and taxonomy of the genus Heteromysis as in Wittmann \& Wirtz (2017); that publication is also used as the model for species descriptions. Larval stages are distinguished according to Wittmann (1981). With certain modifications, as stated by Wittmann (2000), appendage terminology is according to Tattersall \& Tattersall (1951), and for non-sensory cuticle structures according to Klepal \& Kastner (1980). Terminology of gross structures of the foregut follows Kobusch (1998), modified spines of the foregut according to Wittmann \& Griffiths (2018). 
The adjective 'flagelliform' is here used for whip-like structures (Fig. 10G) continuously tapering, without differentiation into a whip handle and whipcord; 'flagellate' for any bifid structures (Fig. 12D) bearing a flagellum in subapical or more proximal position. 'Whip seta' (Fig. 12E, M) is used following Wilson (1989) for setae with a basal part (handle) bearing a thin flagellum (cord, sensory part) at its tip; here, the handle and the cord separated by an articulation, suture, or at least by an optically dense section upon standard microscopy.

\section{Repositories}

NHMW $=$ Natural History Museum of Vienna

USNM = Smithsonian National Museum of Natural History, Washington DC

\section{Abbreviations}

ad. $\quad=$ adult

$\mathrm{BL} \quad=$ body length measured from anterior margin of carapace to posterior margin of telson without spines

$\mathrm{N} 1$ to $\mathrm{N} 4$ = nauplioid larvae at substage N1 freshly hatched from the egg membrane, up to N4 for those shortly before the moult leading to the postnauplioid stage

P1 to P3 = postnauplioid larvae at substage P1 freshly moulted, up to P3 that lasts until moult to juvenile stage upon or shortly after release from brood pouch

\# $\quad=$ sample numbers in Table 1

\section{Results}

Class Malacostraca Latreille, 1802

Order Mysida Boas, 1883

Family Mysidae Haworth, 1825

Subfamily Heteromysinae Norman, 1892

Tribe Heteromysini Norman, 1892

Genus Heteromysis S.I. Smith, 1873

\section{Patterns of modified pleopods in the genus Heteromysis}

Pleopods reduced to small bilobate or obscurely bilobate, setose plates with residual differentiation of pseudobranchial lobes, are among the main diagnostic characters of the genus Heteromysis. The monotypic subgenus Neoheteromysis Băcescu, 1976, is characterized by pleopods 2-4 modified by elongate, smooth setae in both sexes. Modifications of male pleopods by spines or by attenuated setae are typical of the subgenus Olivemysis Băcescu, 1968, where they show a great species-specific diversity related to nine different morphological constellations. Unexpectedly, all four new species of Olivemysis described below are quite unusual by demonstrating a total of three different, rare combinations of pleopods modified by spines. More common modifications are known in six insufficiently known species so far not assigned to any subgenus. Pleopods of both sexes are unmodified (as far as known) unless listed in the following (data from first descriptions of species; additional data sources given in square brackets):

Male Pleopods 1, 3-4. H. (O.) cocoensis Price, Heard \& Vargas, 2018.

Male Pleopods 1-4.H.(O.) gomezi Băcescu, 1970;H.(O.) mayana Brattegard, 1970; H.(O.) smithsoniana sp. nov.

Pleopods 1-5 IN both SeXes. H. (O.) mclellandi Price \& Heard, 2011. 
Male pleopod 2. H. (O.) dardani Wittmann, 2008 (in small adult males only); H. (O.) sixi sp. nov.

Male Pleopods 2-4. H. (O.) dardani Wittmann, 2008 (in average-sized and large males); H. (O.) ekamako Wittmann \& Chevaldonné, 2016; H. (O.) macrophthalma Băcescu, 1983; H. (O.) meenakshiae Bamber, 2000 (pleopod 4 unknown); H. (O.) quadrispinosa Murano, 1988; H. tattersalli H. Nouvel, 1942 [O.S. Tattersall 1967]; H. (O.) tenuispina Murano, 1988; H. (O.) wirtzi Wittmann, 2008; H. (O.) zeylanica W.M. Tattersall, 1922 [in material from Australia according to Murano 1988].

Male Pleopods 2-4, female Pleopod 2. H. (O.) hornimani sp. nov.; H. (O.) waikikensis sp. nov.

Pleopods 2-4 IN Both SEXes. H. (N.) muelleri Băcescu, 1976.

Male Pleopods 2-5, female Pleopod 2. H. (O.) kushimotensis Murano \& Fukuoka, 2003.

Male pleopods 3-4. H. (O.) abrucei Băcescu, 1979; H. (O.) agelas Modlin, 1987; H. (O.) beetoni Modlin, 1984; H. (O.) coralina Modlin, 1987; H. digitata W.M. Tattersall, 1927 [O.S. Tattersall 1967]; H. (O.) domusmaris Wittmann \& Abed-Navandi, 2019; H. (O.) essingtonensis Murano, 1988; H. (O.) guitarti Băcescu, 1968; H. (O.) kensleyi Modlin, 1987; H. (O.) mariani Băcescu, 1970; H. (O.) maxima Murano, 1998; H. (O.) modlini Price \& Heard, 2011; H. odontops Walker, 1898 [O.S. Tattersall 1967]; H. panamaensis O.S. Tattersall, 1967; H. (O.) rubrocincta Băcescu, 1968; H. (O.) sabelliphila Wittmann \& Wirtz, 2017; H. (O.) siciliseta Brattegard, 1970; H. singaporensis O.S. Tattersall, 1967; H. (O.) tuberculospina Modlin, 1987; H. (O.) zeylanica W.M. Tattersall, 1922 [in material from Indian Ocean according to O.S. Tattersall 1967 and Pillai 1968].

Male Pleopod 4. H. (O.) actiniae Clarke, 1955 [Brattegard 1969]; H. (O.) bermudensis G.O. Sars, 1885 [Băcescu \& Iliffe 1986]; H. (O.) ebanksae Price \& Heard, 2008; H. (O.) floridensis Brattegard, 1969 [Modlin 1987]; H. (O.) xanthops Ii, 1964 [O.S. Tattersall 1967]. O.S. Tattersall (1967) claimed modified male pleopods 4 for $H$. gymnura W.M. Tattersall, 1922, but did not state whether she referred to the (meanwhile probably lost) types from the Gulf of Manaar (N Indian Ocean) or to non-types from Zanzibar. According to Wittmann (2013), materials from Zanzibar and Australia attributed to H. gymnura by O.S. Tattersall $(1962,1967)$ and Murano $(1988)$, respectively, do not belong to this species.

Only females known in $H$. (O.) ningaloo Daneliya, 2012 and $H$. (O.) sexspinosa Murano, 1988, each without modified pleopods.

Among the here listed species, female pleopods were so far not described in H. abrucei, H. floridensis, H. gomezi, H. gymnura, H. maxima, H. odontops, H. panamaensis, $H$. sixi sp. nov., H. tattersalli, H. tenuispina, H. tuberculospina, and H. zeylanica.

\section{Subgenus Olivemysis Băcescu, 1968}

Olivemysis Băcescu, 1968: 237 (defined in key).

Olivaemysis - Băcescu 1970: 11-16 (incorrect subsequent spelling); 1981: 85 (evolution). Hanamura \& Kase 2001: 17 (expressed doubt on subgenus concept).

Olivemysis - Bowman \& Orsi 1992: 739 (definition in key). — Bravo \& Murano 1996: 483 (in key). — Wittmann 2008: 368-370 (revalidated spelling, etymology). — Price \& Heard 2011: 43-44 (first formal diagnosis). - San Vicente \& Monniot 2014: 341 (taxonomy, in key).

\section{Type species}

Heteromysis (Olivemysis) rubrocincta Băcescu, 1968, by monotypy. 


\section{Species inventory}

A total of 39 species, including the new ones, is given in the key below. For 30 species see list in Price \& Heard (2011). Nine species of this subgenus described after 2011 are H. cocoensis Price, Heard \& Vargas, 2018; H. domusmaris Wittmann \& Abed-Navandi, 2019; H. ekamako Wittmann \& Chevaldonné, 2017; H. hornimani sp. nov.; H. ningaloo Daneliya, 2012; H. sabelliphila Wittmann \& Wirtz, 2016; H. sixi sp. nov.; H. smithsoniana sp. nov. and H. waikikensis sp. nov.

\section{Definition}

Definition of subgenus modified after Price \& Heard (2011), using present terminology, mainly in order to receive Heteromysis sixi sp. nov.: bifid flagellate spine, usually directed disto-mesially, and long, unbranched seta directed disto-laterally on disto-mesial edge of the antennular trunk; thoracic endopod 3 prehensile, moderately robust, some of the distal articles enlarged; disto-mesial edge of merus 3 not serrated, without tooth-like extension; propodus 3 mostly without paradactylary setae but, if any, representing small, simple setae; some of male pleopods 1-5 (in most species including pleopod 4), if any, modified by flagellate spines (or by attenuated setae); endopod of uropods shorter than exopod. Secondary diagnostic features are present in most species: eyestalks with disto-mesial process (tooth, tubercle), and male thoracic sternites with median processes.

\section{Morphological note}

Twelve out of a total of 35 previously known species here acknowledged as pertaining to this subgenus, have male pleopod 2 and additional pleopods modified. Male pleopod 2 is also modified in the four new species of Heteromysis, including two species with pleopod 2 modified also in females. These four species also share disto-mesial teeth on eyestalks and median processes on male thoracic sternites.

\section{Key to species of the subgenus Olivemysis Băcescu, 1968}

The subgeneric assignment is here explicitly indicated for all 39 acknowledged species of Olivemysis. An additional 13 species given below are insufficiently known and therefore so far not assigned to any subgenus.

1. Lateral margins of telson armed with spines along most of proximal half and beyond 2

- Lateral margins of telson armed with spines only on distal half or at most on distal $3 / 4$

2. Lateral margins of telson armed with continuous series of spines, all along or at least along the stretch reaching from $1 / 5$ to $4 / 5$ distance from the basis (not counting the apical spines) .................. 3

- Lateral margins of telson armed with spines in basal and (sub)-terminal portions except for a smooth stretch in between

3. Telson cleft with laminae along $<4 / 5$ of its margins, distally remaining portion smooth

- Telson cleft with laminae along $\geq 4 / 5$ of its margins.

4. Endopod of uropod with spines only on proximal half

- Endopod of uropod with spines on proximal and on (entire or part of) distal half ......

5. Disto-mesial edge of antennular trunk with inconspicuously flagellate, large, robust spine, 0.7 times length of mesial margin of terminal segment of trunk; endopod of uropod with 5 spines

H. (O.) kensleyi Modlin, 1987 (Gulf of Mexico)

- Disto-mesial edge of antennular trunk with well-flagellate, blade-like spine; blade measured without flagellum $<0.6$ times as long as mesial margin of terminal segment of trunk; endopod of uropod with $<5$ spines 
6. Male pleopods 2-4 with 4-5, 8-14, and 21-28 flagellate spines, respectively, and female pleopod 2 with 2-4 flagellate spines, remaining pleopods with normal setae only; telson cleft with 18-22 laminae.

hornimani sp. nov. (public aquaria in Western to Central Europe: London, Paris, Brest and Wroclaw)

- Pleopod 2 without spines in both sexes; male pleopod 4 with 5-11 modified spines; telson cleft with 10-20 laminae.

7. Male pleopod 3 with 3-5 attenuated spines, male pleopod 4 with 5-6 attenuated spines; thoracic endopod 3 with carpopropodus length $<2$ times width; telson cleft with 10-16 laminae

H. (O.) modlini Price \& Heard, 2011 (Caribbean)

- Male pleopod 3 with 11 flagellate spines, male pleopod 4 with 17 flagellate spines; thoracic endopod 3 with carpopropodus length $>2$ times width; telson cleft with about 20 laminae.

H. (O.) abrucei Băcescu, 1979 (Australia)

8. Endopod of uropods with 5-6 spines; lateral margins of telson with 9-10 spines

H. (O.) guitarti Băcescu, 1968 (Caribbean, Gulf of Mexico)

- Endopod of uropods with 8-15 spines; lateral margins of telson with 18-24 spines.

9. Endopod of uropods with 13-15 spines; lateral margins of telson with 18-21 spines.

H. (O.) essingtonensis Murano, 1988 (Australia)

- Endopod of uropods with 8-10 spines; lateral margins of telson with 20-24 spines.

H. (O.) siciliseta Brattegard, 1970 (Caribbean)

10. Disto-mesial edge of antennular trunk with modified (mostly flagellate) spine in addition to setae

- Disto-mesial edge of antennular trunk without modified spines, with whip setae in addition to normal setae

11. Endopod of uropods with 2 spines.....

- Endopod of uropods with 3-5 spines.

12. Lateral margins of telson with 18-19 spines; telson cleft with 25 laminae

- Lateral margins of telson with 15-16 spines; telson cleft with 12 laminae

H. (O.) agelas Modlin, 1987 (Bahamas)

13. Lateral margins of telson with 10-12 spines; telson cleft with 23-31 laminae; endopod of uropod with 4 spines

H. (O.) quadrispinosa Murano, 1988 (Australia)

- Lateral margins of telson with $>14$ spines

14. Lateral margins of telson with $<19$ spines

- Lateral margins of telson with $\geq 19$ spines

15. Telson cleft with 18-20 laminae; endopod of uropods with 5 spines; flagellate spine on disto-mesial edge of antennular trunk terminally with tubercles

H. (O.) tuberculospina Modlin, 1987 (Caribbean)

- Telson cleft with 22-24 laminae; endopod of uropods with 3-4 spines; flagellate spine on distomesial edge of antennular trunk without tubercles H. waitei W.M. Tattersall, 1927 (Australia)

16. Lateral margins of telson with 19-20 spines; telson cleft with 31 laminae..

H. bredini Brattegard, 1970 (only 1 known) (Caribbean, Gulf of Mexico)

- Lateral margins of telson with 24-25 spines; telson cleft with about 35 laminae.

H. (O.) maxima Murano, 1998 (only 1 đ known) (Australia) 
17. Endopod of uropods with 1 spine; carpopropodus of thoracic endopod 3 with 9-10 strong spines; telson cleft with 16-18 laminae ...............H. panamaensis O.S. Tattersall, 1967 (E Pacific: Panama)

- Endopod of uropods with 4 spines; carpopropodus of thoracic endopod 3 with 3-7 strong spines; telson cleft with 30 laminae

H. odontops Walker, 1898 (NE Pacific)

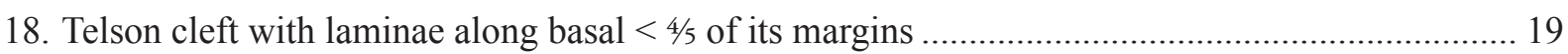

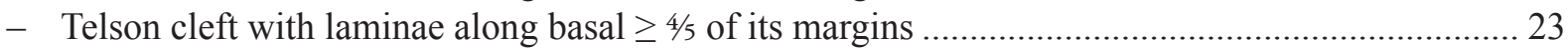

19. Antennal sympod with spiniform extension on outer face; eyestalks with field of scales on mesial margin; basal and median segments of antennular trunk each with dorsal apophysis bearing modified setae (spines) plus normal setae; merus of thoracic endopod 3 with series of 4-6 unilaterally barbed setae on rostral face; only male pleopods 3-4 modified by flagellate spines; endopod of uropods with 2-6 spines along inner margin .....

H. (O.) domusmaris Wittmann \& Abed-Navandi, 2019 (tanks of the Haus des Meeres Aquarium in Vienna, Austria)

- Antennal sympod without or with terminally rounded extension on outer face; basal and median segments of antennular trunk without dorsal apophysis (unknown in H. zeylanica); eyestalks without field of scales on mesial margin.

20. Endopod of uropods with 3-4 spines near statocyst; merus of thoracic endopod 3 without unilaterally barbed setae

- Endopod of uropods with 7-11 spines along inner margin 21

21. Exopod of uropods with modified, sickle-shaped seta subapically on outer margin; merus of thoracic endopod 3 with 4 unilaterally barbed setae on lateral margin

H. disrupta Brattegard, 1970 (only 1 q known) (Caribbean)

- Exopod of uropods with normal setae only; merus of thoracic endopod 3 without unilaterally barbed setae; only male pleopods $3-4$ modified by flagellate spines

H. (O.) zeylanica W.M. Tattersall, 1922 (Indian Ocean, Australia)

22. Thoracic endopods 5-8 with 6-segmented carpopropodus; male pleopods $2-4$ modified by flagellate spines

H. (O.) macrophthalma Băcescu, 1983 (Australia)

- Thoracic endopods 5-8 with 7-segmented carpopropodus

H. (O.) ningaloo Daneliya, 2012 (only 1 q known) (Australia)

23. Endopod of uropods with 2 spines near statocyst; 6 spines on basal third of lateral margins of telson.

H. (O.) sexspinosa Murano, 1988 (Australia)

- Endopod of uropods with 13 spines along inner margin; 2 spines on basal third of lateral margins of telson. H. singaporensis O.S. Tattersall, 1967 (Singapore)

24. Telson cleft with laminae along $\geq 4 / 5$ of its margins

- Telson cleft with laminae along $<4 / 5$ of its margins, distally remaining portion smooth 25

25. Telson elongate with constriction at $2 / 3$ length from basis, telson length $2-3$ times maximum width near basis, lateral margins concave; uropods without spines; telson cleft $16 \%$ telson length; cleft proximally with 2-3 laminae H. filitelsona Modlin, 1984 (Gulf of Mexico)

- Telson without lateral constriction 26

26. Endopod of uropods without spine; carpopropodus of thoracic endopod 3 with 3 strong spines; apical cleft $>1 / 3$ telson length; cleft with about 25 laminae

H. gymnura W.M. Tattersall, 1922 (N Indian Ocean: Gulf of Manaar)

- Endopod of uropods with $>1$ spine 


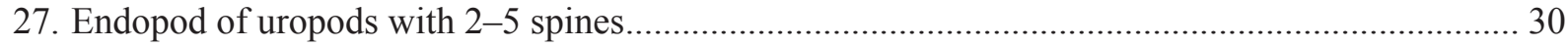

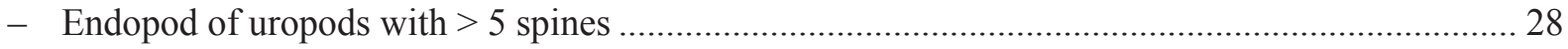

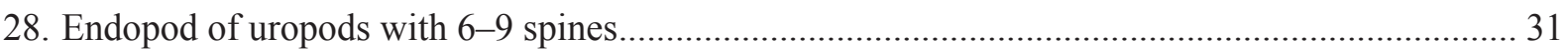

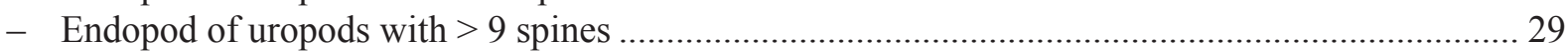

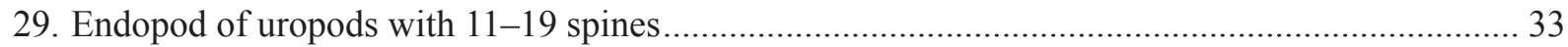

- Endopod of uropods with 40-45 spines; carpopropodus of thoracic endopod 3 with 4 spines; apical cleft $0.2-0.3$ times as long as telson; cleft with 8-13 laminae

H. (O.) actiniae Clarke, 1955 (Caribbean, Bahamas)

30. Endopod of uropods with 2-3 spines near statocyst; carpopropodus of thoracic endopod 3 with 6-7 flagellate spines; male pleopod 2 ending in a large, non-flagellate spine.

H. (O.) ekamako Wittmann \& Chevaldonné, 2016 (Central Pacific: Marquesas)

- Endopod of uropods with 5 spines near statocyst; carpopropodus of thoracic endopod 3 with 8-9 flagellate spines; male pleopod 2 not modified, without spines

H. (O.) coralina Modlin, 1987 (Gulf of Mexico)

31. Endopod of uropods with 6-7 spines on proximal half; apical cleft $1 / 5$ telson length; cleft with $10-14$ laminae; male pleopods 3-4 with 3 and 4 flagellate spines, respectively

H. (O.) mariani Băcescu, 1970 (Caribbean)

- Endopod of uropods with 9 spines on proximal $2 / 3$; apical cleft $3 / 10$ telson length, cleft with $15-17$ laminae; male pleopod 4 with 20-29 flagellate spines

32. Pleopod 2 not modified in both sexes; male pleopod 3 with 6-7 stout, robust, plumose (i.e., not flagellate) setae along distal margin; male pleopod 4 with 20 flagellate spines; carpopropodus of thoracic endopod 3 with 4 pairs of flagellate spines; lateral margins of telson with 9-12 spines ………………………………………....... H. (O.) beetoni Modlin, 1984 (Gulf of Mexico)

- Male pleopod 2 with 3 smooth, tooth-like spines; pleopod 3 with 20 flagellate spines, pleopod 4 with 29 flagellate spines; carpopropodus of thoracic endopod 3 with 6 flagellate spines; lateral margins of telson with 11-13 spine

H. (O.) tenuispina Murano, 1988 (only adult male and immature $q$ known) (Australia)

33. Apical cleft 0.4-0.5 times telson length, cleft with 6-11 laminae; endopod of uropods with 14-19 spines; male pleopod 4 with about 26 flagellate spines

H. (O.) floridensis Brattegard, 1969 (Gulf of Mexico, Bahamas)

- Apical cleft 0.2-0.3 times telson length; lateral margins of telson with 5-9 spines; only male pleopod 4 modified

34. Male pleopod 4 with 2-9 flagellate spines; telson cleft with 6-10 laminae; carpopropodus of thoracic endopod 3 with 5-6 spines

H. (O.) ebanksae Price \& Heard, 2008 (Caribbean)

- Male pleopod 4 with > 25 spines; telson cleft with 11-20 laminae; carpopropodus of thoracic endopod 3 with 6-7 flagellate spines.

H. (O.) bermudensis G.O. Sars, 1885 (35)

35. Telson cleft with 15-20 laminae; distal margin of male pleopod 4 with about 35-55 flagellate spines .............................H. (O.) bermudensis bermudensis G.O. Sars, 1885 (Bermuda, Caribbean)

- Telson cleft with 14 laminae; distal margin of male pleopod 4 with 26-30 normal, non-flagellate spines ………………….....H. (O.) bermudensis cesari Băcescu, 1968 (Gulf of Mexico, Caribbean)

36. Merus of thoracic endopod 3 with about 8 finger-like processes proximally on mesial margin ......... H. digitata W.M. Tattersall, 1927 (Red Sea)

- Merus of thoracic endopod 3 without finger-like processes 
37. Distal half of endopod of uropods without spines.

- Endopod of uropods with 14-15 spines reaching from proximal to at least part of distal half; male pleopod 4 modified by 7 small, flagellate spines; carpopropodus of thoracic endopod 3 with 3 flagellate spines

H. (O.) xanthops Ii, 1964 (Japan)

38. Endopod of uropods without spine; pleopods without spines in both sexes

- Endopod of uropods with at least one spine.....

39. Endopod of uropods with one spine

- Endopod of uropods with more than one spine

40. Lateral margins of telson with 5 spines; telson cleft with 16-17 laminae; carpopropodus of thoracic endopod 3 with 3 flagellate spines

H. dentata Hanamura \& Kase, 2001 (E Indian Ocean: Christmas Island)

- Lateral margins of telson with $>6$ spines; telson cleft with $>18$ laminae 41

41. Lateral margins of telson with 7-11 spines; telson cleft with 28-32 laminae

H. dispar Brattegard, 1970 (Gulf of Mexico)

- Lateral margins of telson with 10-12 spines; telson cleft with 19-24 laminae

H. komaii Fukuoka, 2005 (Japan)

42. Male pleopods 2-4 modified by flagellate spines, male pleopod 5 modified by about 11 short setae on apical to disto-mesial margin; all female pleopods without spines, with normal setae only; lateral margins of telson with 5-9 spines; telson cleft with about 22-31 laminae .....H. (O.) kushimotensis Murano \& Fukuoka, 2003 (Aquarium of the Kushimoto Marine Park Center, Japan)

- At least pleopod 5 unmodified in both sexes; lateral margins of telson with 6-10 spines 43

43. Male pleopod 2 modified by flagellate spines; remaining male pleopods not modified; lateral margins of telson with 6 spines; telson cleft with 26 laminae

H. (O.) sixi sp. nov. (only 1 त known) (tanks of the 'Waikiki Aquarium', Hawaii)

- Male pleopod 2 variously modified; male pleopod 3 modified by flagellate spines

44. Pleopod 2 with 5-6 minute flagellate spines in both sexes; male pleopods $3-4$ with 2-7 and 11-12 flagellate spines, respectively; remaining pleopods without spines in both sexes; lateral margins of telson with 8-10 spines; telson cleft with 25-32 laminae

H. (O.) waikikensis sp. nov. (tanks of the 'Waikiki Aquarium', Hawaii)

- Male pleopod 2 variously modified but not with flagellate spines; male pleopod 3 with 13-22 flagellate spines; all female pleopods normal

45. Male pleopod 2 tapering to form a seta-like apical process; male pleopod 3 with 15 flagellate spines; lateral margins of telson with 10 spines; telson cleft with about 30 laminae......

.H. (O.) meenakshiae Bamber, 2000 (W Pacific: Hong Kong)

- Male pleopods 1-2 ending in a large, smooth spine (stylet); male pleopod 3 with 13-22 flagellate spines; lateral margins of telson with 6-9 spines; telson cleft with about 14-22 laminae 50

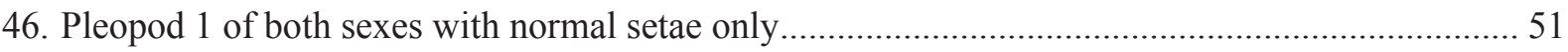

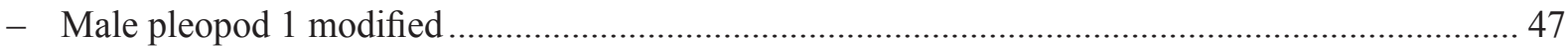

47. Male pleopod 1 with 6 smooth, robust spines, pleopod 3 with 5-6 flagellate spines, pleopod 4 with dense series of 28-36 small blunt spines; male pleopods 2, 5, and all female pleopods without 
spines; telson cleft with 16-21 laminae.....

H. (O.) cocoensis Price, Heard \& Vargas, 2018 (equatorial E Pacific: Coco Island)

- Male pleopod 1 ends in only one smooth spine or in attenuated setae.

48. All pleopods non-dimorphic, with 14-29 modified attenuated setae in both sexes; rostrum triangular, apically broadly rounded, about half the length of terminal segment of antennular trunk; telson cleft with 12-15 laminae......

H. (O.) mclellandi Price \& Heard, 2011 (Caribbean)

- Male pleopods 1-2 ending in a long, smooth spine (stylet), male pleopods 3-4 modified by flagellate spines; male pleopod 5 and all female pleopods with normal setae only

49. Antennal scale and carpopropodus of thoracic endopod 3 subdivided; carpopropodus of thoracic endopod 3 with 6 flagellate spines.

H. (O.) smithsoniana sp. nov. (tanks of the 'Smithsonian Marine Ecosystems Aquarium', Florida)

- Antennal scale undivided; carpopropodus of thoracic endopod 3 undivided, with 3-5 flagellate spines

50. Rostrum $<1 / 4$ length of terminal segment of antennular trunk; male pleopods 3-4 with 15 and 18 flagellate spines, respectively; telson cleft with 20-22 laminae; endopod of uropods with 3-2 spines

H. (O.) gomezi Băcescu, 1970 (Caribbean)

- Rostrum > 4/5 length of terminal segment of antennular trunk; male pleopods 3-4 with 13-22 and 19-35 flagellate spines, respectively; telson cleft with 14-20 laminae; endopod of uropods with 1-4 spines

H. (O.) mayana Brattegard, 1970 (Caribbean, Gulf of Mexico)

51. Lateral margins of telson with 10-11 spines; rostrum about half as long as terminal segment of antennular trunk; male pleopods 3-4 with 6-10, and 8-15 flagellate spines, respectively; male pleopods $1-2,5$ and all female pleopods without spines; endopod of uropods with 4-5 spines H. (O.) rubrocincta Băcescu, 1968 (Gulf of Mexico)

- Lateral margins of telson with 4-9 spines; rostrum exceeds half the length of terminal segment of antennular trunk.

52. Male pleopod 3 with 7-8 large flagellate spines, pleopod 4 with 7-11 much smaller flagellate spines; male pleopods $1-2,5$, and all female pleopods not modified; rostrum $80-90 \%$ as long as terminal segment of antennular trunk; telson cleft with 15-22 laminae; endopod of uropods with 2-3 spines

H. (O.) sabelliphila Wittmann \& Wirtz, 2017 (NE Atlantic: Cape Verde Islands)

- Male pleopod 2, and mostly also pleopods 3-4 modified; male pleopods 1,5 and all female pleopods not modified; telson cleft with 21-27 laminae; endopod of uropods with 3-4 spines

53. Antennular trunk with two forwardly directed, smooth setae on disto-mesial edge of terminal segment; no flagellate spine present. Apical cleft 22-23\% telson length

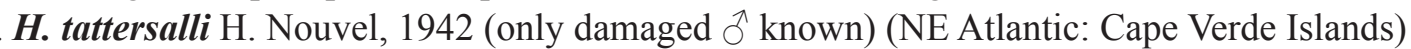

- One strong, obliquely anteriorly directed, flagellate spine plus one large, smooth, antero-laterally directed seta on disto-mesial edge of terminal segment of antennular trunk. Apical cleft 24-29\% telson length.

54. Male pleopods $2-4$ with $1-7,0-4$, and $0-4$ flagellate spines, respectively; endopod of uropods with 3 spines; telson cleft with 21-23 laminae.

H. (O.) dardani Wittmann, 2008 (NE Atlantic: Madeira)

- Male pleopods 2-4 with 9, 16-17, and 19 mostly flagellate spines, respectively; endopod of uropods with 4 spines; telson cleft with 24-27 laminae

H. (O.) wirtzi Wittmann, 2008 (NE Atlantic: Madeira) 


\section{Heteromysis (Olivemysis) smithsoniana sp. nov. urn:1sid:zoobank.org:act:4B3ABD3F-EF26-4C69-B951-C81B6FE5DCFE}

Figs $1-3$

\section{Diagnosis}

Rostrum (Figs 1C, 2F) forms a triangular plate between the eyes, rostrum 58-83\% length of terminal segment of antennular trunk; apex acute to narrowly rounded. Disto-mesial edge of eyestalks with welldeveloped tooth (Fig. 1C). Cornea occupies distal $25-30 \%$ of eye surface. Antennular trunk (Fig. 2A-C) with strong, subapically flagellate spines in addition to setae, namely one spine on dorsal apophysis of basal segment and a somewhat larger one on disto-mesial edge of terminal segment. Antennal scale (Fig. 2E) with small apical segment; antennal scale reaches $0-20 \%$ of its length beyond antennal peduncle. Scale length is 3.0-3.3 times maximum width. Male thoracic sternites 1-8 (Fig. 1D) each with mid-ventral process. Female sternites without processes. Both sexes with flagellum of thoracic exopods 1-8 showing 8, 9, 9, 9, 9, 9, 9-10, and 9-8 segments, respectively (Fig. 3F). Carpopropodus of thoracic endopods $1-8$ with 2, 2, 2, 4-3, 7, 7, 7, and 7 segments, respectively (Figs $2 \mathrm{~L}, \mathrm{O}, 3 \mathrm{C}, \mathrm{F}$ ). No visible difference between left and right thoracic endopods 3. Shape of carpopropodus 3 sexually dimorphic (Figs 2L vs 2O), length 2.1-2.7 times maximum width in males vs 2.9-3.4 in females. Disto-mesial edge of merus 3 without tooth-like extension. Carpus and propodus separated by a distinct suture. Carpus with six flagellate spines (Fig. 2M, P) on distal $45-55 \%$ of inner margin in both sexes. Propodus without paradactylary setae and without spines. Ischium of endopod 5 without excavation. Penes $58-67 \%$ length of ischium of endopod 8; proximal $2 / 3$ tube-like, distal $1 / 3$ narrowing (Fig. 3F). Pleopods rudimentary, unsegmented, with residual differentiation of endopod (pseudobranchial lobe). All female pleopods and male pleopod 5 without spines. Pleopods 1-4 dimorphic (Fig. 3H-L vs 3O): male pleopods 1-2 end with a long smooth spine (stylet); distal half of pleopods 3-4 knife-shaped, with broadly rounded terminal margin; pleopod 3 with 25-30 small, flagellate spines, densely set along 'blade'; pleopod 4 with 28-33 spines of same structure and size; flagella same-sized or longer than spines. Exopod of uropods 1.1-1.4 times length of endopod (Fig. 1E); endopod with 3 (rarely 2) spines on inner margin near statocyst; distal spine-free portion 55-62\% length of endopod. Proximal 21-33\% of lateral margins of telson without spines, distal portion with 7-11 spines. Telson (Fig. 3R) with U- to V-shaped apical cleft penetrating $29-36 \%$ telson length; cleft with $22-30$ laminae along basal $75-86 \%$ of its margins. Apical lobes of telson each with two spines; latero-apical spines are $14-18 \%$ telson length; medio-apical spines are $0.5-0.6$ times length of latero-apical spines.

\section{Etymology}

The species name is a Latinized adjective with female ending, referring to the Smithsonian Marine Ecosystems Aquarium (Fort Pierce, Florida).

\section{Type material}

\section{Holotype}

FLORIDA • $\widehat{O}^{\Uparrow}$ ad., BL $3.6 \mathrm{~mm}$ (in vial); Fort Pierce, Smithsonian Marine Ecosystems Aquarium; Dec. 2019; Bill Hoffman leg.; NHMW 26968.

\section{Paratypes}

FLORIDA -3 q $q$ ad., BL $3.3-4.0 \mathrm{~mm}, 2$ ô $\widehat{\text { ad }}$., BL 3.6-3.8 $\mathrm{mm}$ (in vial); same collection data as

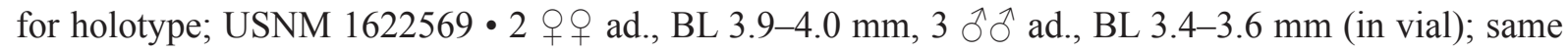
collection data as for holotype; NHMW $26969 \cdot 1$ q ad., BL $4.2 \mathrm{~mm}$ (on slides); same collection data as for holotype; NHMW $26970 \bullet 1$ ad., BL $4.8 \mathrm{~mm}$ (on slides); same collection data as for holotype; NHMW 26971 • 1 § ad., BL 4.2 mm (on slides); same collection data as for holotype; NHMW 26972. 

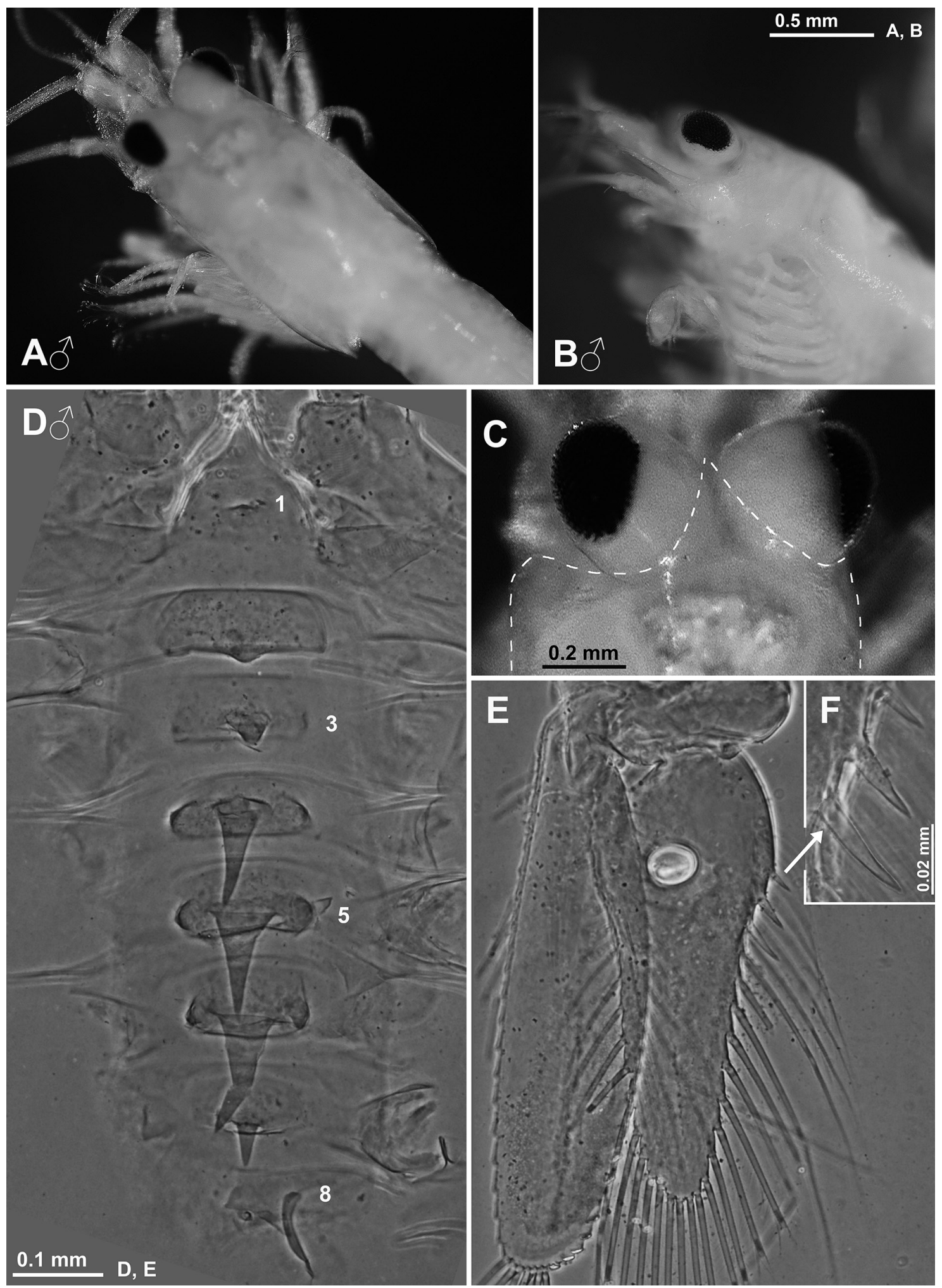

Fig. 1. Heteromysis smithsoniana sp. nov. A-C. Holotype, ô with body length $3.6 \mathrm{~mm}$ (NHMW 26968). D-F. Paratype, ơ $4.8 \mathrm{~mm}$ (NHMW 26971). A. Cephalothorax, dorsal. B. Cephalothorax, lateral. C. Head region, obliquely dorsal; dashed line illustrates anterior contour of carapace. D. Thoracic sternites expanded on slide, ventral. E. Uropods, dorsal. F. Detail of uropods (E) showing spines of endopod. A-F. Ethanol-fixed materials on petri dishes (A-C) and slides (D-F). 


\section{Description}

All features of the Diagnosis. Size of adult females 3.3-4.2 mm, males 3.4-4.8 mm. Cephalothorax comprises $22-33 \%$ body length, pleon (without telson) $50-65 \%$, carapace (without rostrum) $20-25 \%$, and rostrum 4-5\%. Abdominal somites $1-5$ measure $0.6-0.8,0.6-0.9,0.6-0.8,0.5-0.9$, and $0.6-0.9$ times the length of somite 6 , respectively.

CARAPACE (Fig. 2F-H). Carapace covers $65-87 \%$ of cephalothorax dorsally. Rostrum reaches at most to distal $2 / 3$ of artificially straight forward-oriented eyestalks (without cornea). Cervical and cardial sulcus short but distinct. Posterior margin leaving $0.8-1.5$ posterior thoracic somites mid-dorsally exposed. Cervical pore group formed by $8-11$ pores each with about $1 \mu \mathrm{m}$ diameter arranged in a wide arc (Fig. 2G). The posterior group of pores (Fig. 2H) closely behind cardial sulcus; it consists of 10-12 such pores, each with about $1.2 \mu \mathrm{m}$ diameter surrounding a larger but indistinct, rounded structure.

EYES (Fig. 1A-C). Eyestalks and cornea dorsoventrally strongly compressed. Eyestalks without scales. In dorsal view the cornea appears calotte-shaped, measuring 0.6-0.9 times the length of eyestalk (cornea not included). In lateral view (Fig. 1B) the cornea appears kidney-shaped, length 1.6 times maximum width.

Antennulae (Fig. 2A-D). Trunk extends $46-61 \%$ its length beyond eyes, $15-33 \%$ beyond antennal scale. Measured along dorsal midline, the basal segment is $32-47 \%$ trunk length, median segment $12-$ $18 \%$, and terminal segment $38-49 \%$. Basal segment on basal half of its outer face with two small, stout, barbed setae (Fig. 2D). Its dorsal apophysis with 3-2 barbed setae, one flagelliform seta, and one flagellate spine (Fig. 2C). Lateral apophysis with one plumose and one shorter barbed seta, and one flagelliform seta. Median segment dorsally with large apophysis with 3-4 barbed plus one flagelliform seta. Terminal segment 1.4-1.5 times as long as wide. Its mid-dorsal apophysis with 4-2 barbed setae. Disto-mesial edge of terminal segment with flagellate spine (Fig. 2B) resembling certain flagellate spines (Fig. 2P) of gnathopods. In both sexes the outer antennular flagellum is thicker than the inner one by a factor of 1.5-2.3 when measured near basis of flagella. Male lobe well setose, inserts ventrally close to terminal margin of antennular trunk, almost spherical, length is $20-24 \%$ width of terminal segment of trunk, its width $19-26 \%$.

Antennae (Fig. 2E). Three-segmented antennal peduncle: basal segment is $18-23 \%$ peduncle length, second segment $43-49 \%$, and third segment $29-32 \%$. Sympod with tongue-like process on ventral face.

MouthParTs (Fig. 2I-K). Labrum not produced into a spiniform process; caudally with small, stiff bristles, fields of setae on caudal and ventral faces. Mandibular palp (Fig. 2I) three-segmented. Its proximal segment without setae, $10-15 \%$ length of palp. Length of median segment 2.1-2.3 times its maximum width and $65-72 \%$ palp length. Inner margin of median segment with two smooth setae in subapical and subbasal position, respectively, and with 6-7 modified setae along proximal half in both sexes. These setae increasing in length distally; their basal half thickened, bearing unilateral series of stiff barbs; distal half with smooth flagellum (Fig. 2K). Mid-caudal face with 0-1 weakly barbed seta, outer margin with smooth setae all along. Rostral face only in males with longitudinal series of scales near inner margin from subbasal to subterminal portions; scales triangular, facing distally, arranged in groups formed by mostly 7-9 peripheral scales around a central scale (Fig. 2J). Terminal segment strongly setose, $17-21 \%$ palp length. Pars molaris with well-developed grinding surface in both mandibles. Pars incisiva with 4-5 teeth, digitus mobilis with 3-4 teeth, and pars centralis with 3-4 spiny teeth. Labium normal, comprising two hairy lobes with dense set of stiff bristles on distal half of inner face. Maxillula as normal in this genus; distal segment terminally with 11-12 mostly smooth, in part weakly serrated spines; subterminally with three setae barbed on their distal half; transverse series of three pores closely adjoining the outer (= most ventral) seta. Endite of maxillula terminally with three strong, distally spiny 


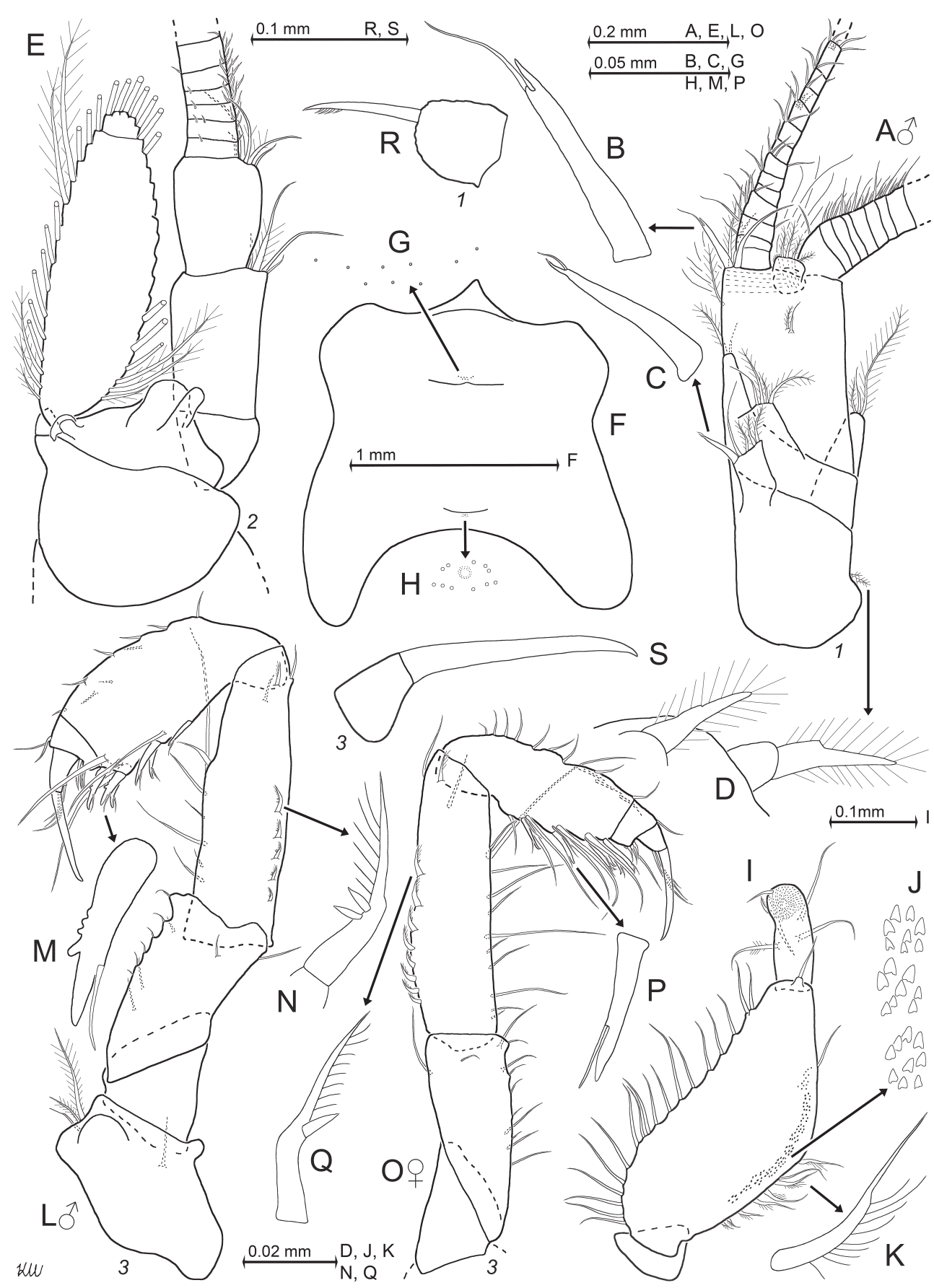

Fig. 2. Heteromysis smithsoniana sp. nov., paratypes, $\widehat{\partial} \hat{\mathrm{o}}$ with body length $4.8 \mathrm{~mm}(\mathrm{~A}-\mathrm{D}, \mathrm{I}-\mathrm{K}, \mathrm{R}-\mathrm{S}$ : NHMW 26971), $4.2 \mathrm{~mm}$ (E-H, L-N: NHMW 26972), and $94.2 \mathrm{~mm}(\mathrm{O}-\mathrm{Q}:$ NHMW 26970). A. Male antennula, dorsal. B-D. Details of antennula (A) showing modified spines (B-C) and setae (D). E. Antenna with antennal gland, dorsal. F. Carapace expanded on slide. G-H. Details of carapace (F) showing pore groups in front of cervical sulcus $(G)$ and behind cardial sulcus (H). I. Palp of left mandible, rostral. J-K. Details of mandibular palp (I) showing three scale groups (J) and one modified seta $(\mathrm{K})$ from inner margin of median segment; most setae of terminal segment are below drawing plane. L. Right male thoracic sympod and endopod 3, rostral. M-N. Details of male endopod 3 (L) showing modified spine $(\mathrm{M})$ of carpus and modified seta $(\mathrm{N})$ of merus. $\mathbf{O}$. Left female thoracic endopod 3, rostral. P-Q. Details of female endopod $3(\mathrm{O})$ showing modified spine $(\mathrm{P})$ of carpus and modified seta $(\mathrm{Q})$ of merus. R-S. Dactyli 1 and 3, with claw, setae omitted. 
setae accompanied by 3-4 proximally thick, barbed setae; inner and outer margins with numerous less thick setae. Maxilla as normal in this genus; terminal segment of endopod with maximum width 53$66 \%$ length. Outer margin of exopod all along with 16-19 mostly plumose setae, the two apical setae larger than the remaining ones.

Male thoracic sternites (Fig. 1D). Median processes of sternites 2-8 emerge from socket-like elevations. Some intermediate sternites with additional, small submedian processes not fused together with median processes. Size increases from processes 1 to 6 , is smaller in process 7, and then increases again to 8 . Processes 1-3 terminally rounded, processes 4-7 acute-angled with acute tip.

Thoracopods (general; Figs 2L-S, 3C-G). Length of flagella as well as of basal plates increases from exopod 1 to 6, and decreases from exopod 6 to 8. Basal plates (Fig. 3F) expanded, 1.7-2.3 times as long as wide in both sexes. Outer margin of the plates ends in a well-rounded edge. First thoracopods with large, leaf-like, smooth epipod. As in many species of this genus, a plumose seta plus a shorter barbed seta present at the intersegmental joint connecting sympod of thoracopod 2 with corresponding thoracic sternite; no such setae in remaining thoracopods. Length of ischium and total length of endopod increase in series of thoracopods 1, 2, 4, 3, 5-8. Basis of endopods 4-8 (Fig. 3F) with a small, lappet-like apophysis on rostral face below endopod. Ischium becomes more slender from endopods 1 to 5 , and its shape remains subequal among endopods 5-8. Ischium shorter than merus in endopods $1-4$ (Fig. 2L, O), but longer than merus in endopods 5-8 (Fig. 3F). Thoracic endopods with claw 3 longest; claws 1, 4-8 (Figs 2R, 3D-E, G) about half as long as claw 3; claw 2 not detected (presence not excluded). Claws 1, 5-8 subapically unilaterally serrated (Figs 2R, 3E, G), claws 3-4 smooth (Figs 2S, 3D). Claw 4 straight (Fig. 3D), claws 1, 3 weakly bent (Fig. 2R-S), claws 5-8 strongly bent (Fig. 3E, G). When stretched anteriorly, endopod 8 reaches to maxillula, when stretched posteriorly to end of pleonite 5 . Penes with small subapical excavation, tip trilobate; no setae (Fig. 3F).

MAXILLIPEDS. Coxa of first maxilliped with small endite bearing one barbed seta at tip. Basis with large, prominent endite that is densely setose on inner margin. Ischium and merus each with one smaller but distinct, medially setose endite. Basis of second maxilliped with large, distinctly medially projecting endite. In both sexes, combined praeischium plus ischium are 0.7-0.9 times length of merus, carpopropodus plus dactylus 1.0-1.2 times merus. Dactylus very large, with dense brush formed by large numbers of normal setae and 10-12 modified setae, the latter apically bent, bearing two symmetrical series of denticles (stiff barbs) on either side in subbasal to median portions.

GNathopods (thoracic endopods 3; Fig. 2L-Q, S). Ischium 1.5-2.0 as long as wide; merus 2.3-3.8 as long as wide and 1.5-1.7 length of ischium. Carpus 0.6-0.8 times length of merus, 1.1-1.3 times ischium. Claw 2.4-4.0 times length of dactylus and $41-43 \%$ of carpopropodus. Distal third of ischium with 3-7 short whip setae on mesial margin, each whip seta on tip of short tooth-like projection (Fig. 2L) in males, the latter projection shorter in females (Fig. 2O). Series of 2-5 unilaterally barbed setae in males (Fig. 2N), 6-9 in females (Fig. 2Q), on basal 50-60\% of outer face of merus, plus single seta in subterminal position. These setae with series of normal barbs (cilia) along median to subapical portions, basal portions thickened. Subterminal seta without, remaining setae at end of thickened portion with 1-2 modified, spine-like barbs; mainly two such 'spines' in males (Fig. 2N), only one in females (Fig. 2Q). Proximal $60-80 \%$ of merus with 3-7 short whip setae on mesial margin, whip setae alternating with longer smooth setae; no tooth-like projections. Carpus with flagellate spines (Fig. 2M, P) arranged as two single spines and more distally two pairs of spines; all these spines with subapical flagellum positioned on smooth posterior (proximal) face; four distal spines rugged to various degrees on anterior face.

MARsupium. Oostegites 1-2 (derivates of thoracopods 7-8) without setae on upper (dorsal) margins. Lower margins from subbasal region up to the rounded tip bearing series of setae, most of which are 


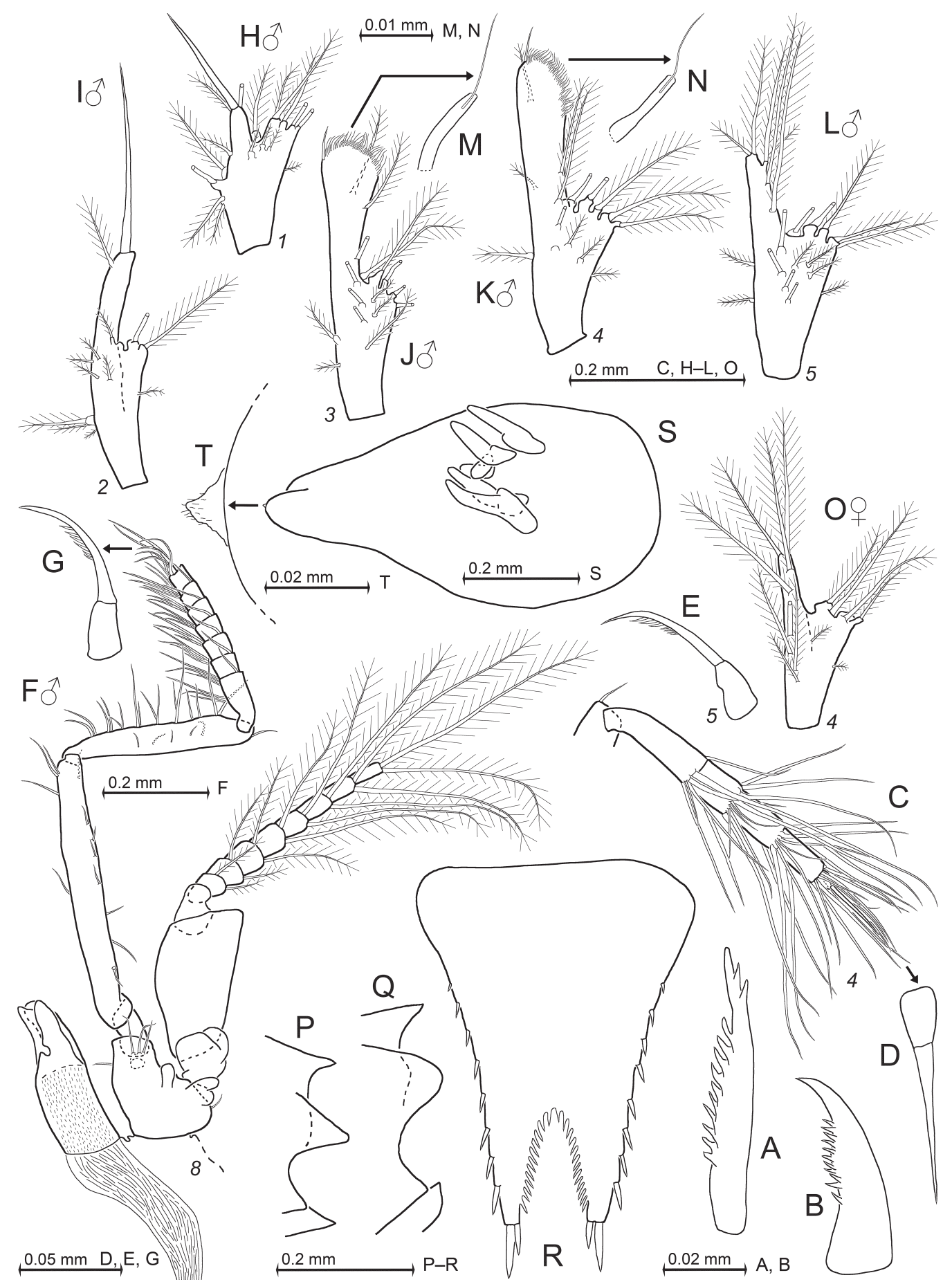

Fig. 3. Heteromysis smithsoniana sp. nov., holotype, ô with body length $3.6 \mathrm{~mm}$ (P: NHMW 26968)

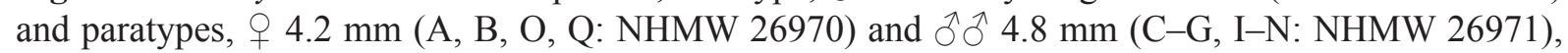
$4.2 \mathrm{~mm}$ (H, R: NHMW 26972). A-B. Modified spines from dorsolateral infoldings (A) and lateralia (B) of foregut. C. Tarsus of thoracic endopod 4, caudal. D. Detail of tarsus 4 (C) showing dactylus with nail. E. Dactylus 5 with nail. F. Thoracopod 8 with penis, rostral. G. Detail of endopod 8 (F) showing dactylus with nail. H-L. Series of male pleopods $1-5$, rostral. $\mathbf{M}-\mathbf{N}$. Details of male pleopods $3(J)$ and 4 (K) showing flagellate spines. O. Female pleopod 4, rostral. P-Q. Variants of scutellum paracaudale, lateral. R. Telson. S. Nauplioid larva at substage N1, ventral. T. Detail of nauplioid larva (S) showing tip of abdomen. 
barbed by fine cilia along subbasal to median portions. Oostegite 1 near basis with 7-10 setae, oostegite 2 with 2-4 setae which are microserrated along distal half. Ventral and rostral portions of outer face of only second oostegite with total of 7-11 slender whip setae, but shorter than barbed setae. Thoracopod 6 with rudimentary oostegite represented by small, rounded lobe terminally with two setae which are microserrated on distal half.

Pleopods (Fig. 3H-O). Female pleopods increase in length caudally. Length of male pleopods increases in series of pleopods $1,2 \approx 5,3,4$. For presence and numbers of spines on male pleopods $1-4$, see 'Diagnosis' above. Stylet of pleopod 1 shorter than that of pleopod 2. Single smooth seta at outer terminal edge of male pleopods 3-4; all other setae of pleopods 1-5 are plumose or barbed in both sexes (not counting flagellate spines).

TAIL FAN (Figs 1E-F, 3P-R). Scutellum paracaudale triangular, tip acute or narrowly (Fig. 3P) to broadly (Fig. 3Q) rounded. Exopod of uropods extends by $12-23 \%$ its length beyond endopod, or $26-48 \%$ beyond telson; endopod $11-30 \%$ its length beyond telson. Diameter of fluorite statoliths is $44-59 \mu \mathrm{m}$ ( $n=9$ statoliths from six specimens). Statoliths discoidal with shallow fundus and distinct tegmen. Statolith formula not available in this material. Telson length 1.2-1.3 times its maximum width or 1.01.3 times length of sixth pleonite. For further details of telson, see 'Diagnosis' above.

Foregut (Fig. 3A-B). Gross structure as described by Wittmann \& Abed-Navandi (2019) for H. domusmaris. Lateralia anteriorly with apically coronate spines with smooth shaft and long spines armed with loose series of small denticles along shaft. Lateralia more caudally with separate group of 4-6 centrally, unilaterally serrated spines (Fig. 3B). Dorsolateral infoldings with two longer, apically pronged spines furnished with series of acute teeth (Fig. 3A) along subbasal to subapical portions. Gut contents of three examined $H$. smithsoniana sp. nov. were mostly unidentifiable material, minor amounts of crustacean remains and even fewer mineral particles.

\section{Distribution}

The species is so far known only from tanks in the 'Smithsonian Marine Ecosystems Aquarium', Fort Pierce, Florida. Origin most likely in coastal marine waters of the subtropical NW-Atlantic (Table 1).

\section{Larvae}

In the ethanol-fixed material, three out of six adult females examined had 1-2 nauplioid larvae in the brood pouch; three lacked a brood. A female with $4.2 \mathrm{~mm}$ body length carried two nauplioid larvae at substage N1 with $0.66-0.69 \mathrm{~mm}$, a female of $3.9 \mathrm{~mm}$ had one N3 with $0.78 \mathrm{~mm}$, and a female of $3.6 \mathrm{~mm}$ had one N4 with $0.84 \mathrm{~mm}$. Larvae at substages N3 and N4 with smooth cuticle all around. The two N1 larvae (Fig. 3S) also with smooth cuticle except for minute down-like structures (Fig. 3T) at tip of abdomen. Remaining features in Fig. 3S are typical of the state of development.

Heteromysis (Olivemysis) hornimani sp. nov. urn:1sid:zoobank.org:act:5C14C755-FE9E-4FD0-9113-F587163584BB

Figs $4-8$

\section{Diagnosis}

Rostrum triangular (Fig. 4A), 69-74\% length of terminal segment of antennular trunk, apex acute to narrowly rounded. Cornea occupies distal third of eye surface. Disto-mesial edge of eyestalks with small tooth (Fig. 4C). Antennular trunk (Fig. 5B-E) with one strong, apically barbed, spine-like seta on dorsal apophysis of basal segment, and with two subapically flagellate spines in addition to setae, namely one spine on disto-mesial edge of median segment, and a broader one on disto-mesial edge of 
terminal segment. Antennal scale (Fig. 5G) with small apical segment; total scale length is 3.0-3.7 times maximum width; antennal scale reaches $0-20 \%$ its length beyond antennal peduncle. Male thoracic sternites 1-8 (Fig. 4D) each with single mid-ventral process. Female sternites without such processes. Flagellum of thoracic exopods 1 and 8 with 8-9 segments, remaining exopods with 9 segments in both sexes (Fig. 6F). Carpopropodus of thoracic endopods $1-8$ with 2, 2, 2, 4 (rarely 5), 5-8, 5-8, 7-9, and 7-9 segments, respectively. No visible difference between left and right thoracic endopods 3. Distomesial edge of merus 3 without tooth-like extension. Carpus 3 separated from propodus by a distinct suture. Length of carpopropodus 3 is 1.8-2.6 times maximum width in males (Fig. 5L), 2.1-3.1 in females (Fig. 5H). Carpus 3 with 6-7 flagellate spines (Fig. 5I-J) along distal 55-78\% of inner (mesial) margin in both sexes; propodus without paradactylary setae or spines. Penes tube-like, $73-94 \%$ length of ischium of endopod 8 (Fig. 6F). Pleopods (Fig. 7) rudimentary, unsegmented, with residual differentiation of endopod (pseudobranchial lobe). Female pleopods 1, 3-5, and male pleopods 1, 5 without spines. Pleopods 3-4 with clearly dimorphic structure, while only spine numbers differ between sexes in pleopod 2. Apical third of female pleopod 2 with series of 2-4 mostly robust, flagellate spines increasing in length distally. Male pleopod 2 with 4-5 robust spines in comparable sequence (i.e., increasing in length distally) and position (i.e., on apical third). Distal half of male pleopods 3-4 knife-shaped with obliquely truncate, somewhat convex terminal margin; pleopod 3 with 8-14 smaller (compared with pleopod 2), flagellate spines, densely set along the 'blade'; pleopod 4 with 21-28 even smaller, flagellate spines along the 'blade'. Exopod of uropods 1.2-1.4 times length of endopod (Fig. 4F); endopod with 2-3 spines near statocyst; distal spine-free portion is $65-71 \%$ length of endopod. Lateral margins of telson (Fig. 6I) all along with 16-20 spines in continuous series; U- to V-shaped apical cleft penetrates telson length by $19-27 \%$; cleft with 18-22 laminae along basal $67-86 \%$ of its margins. Apical lobes of telson each with two spines; latero-apical spines are $12-15 \%$ telson length; medio-apical spines are $0.4-0.6$ times length of latero-apical spines.

\section{Etymology}

The species name is a masculine noun in the genitive singular, referring to the Horniman Museum Aquarium, London.

\section{Material examined}

\section{Holotype}

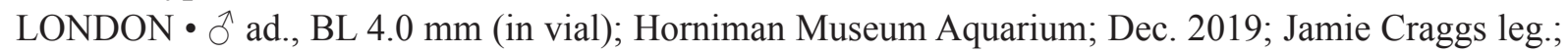
NHMW 26973.

\section{Paratypes}

LONDON 111 $\widehat{\partial}$ ad., BL 3.7-5.4 mm, 3 우 ad., BL 4.7-6.1 mm, 11 subad., 5 imm., 1 juv. (in vial); same collection data as for holotype; NHMW 26974 1 ㅇ ad., BL $5.2 \mathrm{~mm}$ (on slides); same collection data as for holotype; NHMW $26975 \cdot 1$ o ad., BL $4.7 \mathrm{~mm}$ (on slides); same collection data as for holotype; NHMW 26976.

\section{Non-types}

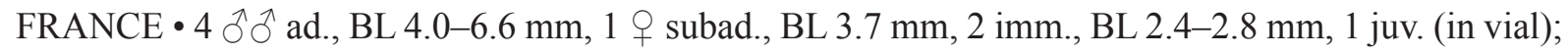
Aquarium de Paris; Sep. 2020; Anaïs Courtet leg.; NHMW 26977.

FRANCE $\bullet 1$ त ad., BL 5.3 mm, 1 imm. (in vial); Brest, Oceanopolis Aquarium; 3 Feb. 2020; Dominique Barthelmy leg.; NHMW 26978 1 q ad., BL 4.3 mm, 1 ô ad., BL 5.4 mm (in vial); same collection data as for preceding; NHMW 26979 • 1 $\widehat{\text { ad., BL }} 6.3 \mathrm{~mm}$ (on slides); same collection data as for preceding; NHMW 26980. 


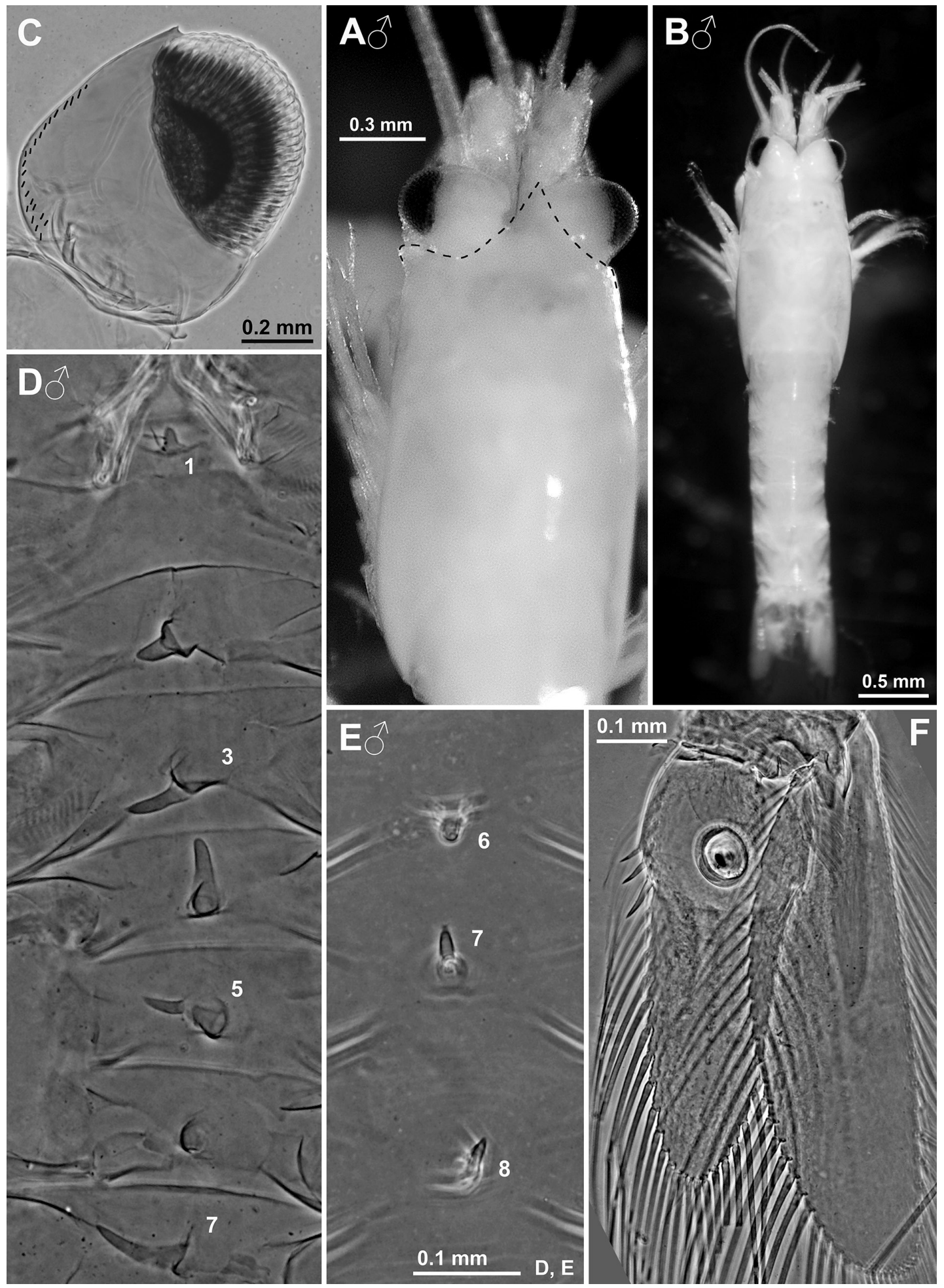

Fig. 4. Heteromysis hornimani sp. nov., holotype, $\widehat{\jmath}$ with body length $4.0 \mathrm{~mm}$ (A: NHMW 26973) and

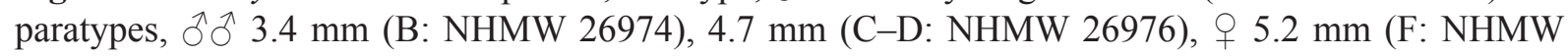
26975), and non-type, $\widehat{\jmath} 6.3 \mathrm{~mm}$ (E: NHMW 26980). A. Cephalothorax, slightly oblique dorsal view; dashed line illustrates the anterior contour of carapace. B. $\hat{\sigma}$ in toto, dorsal view. C. Left eye, ventral; contrast of scales on mesial margin of eyestalk is enhanced with black ink. D. Thoracic sternites 1-7 expanded on slide, ventral. E. Sternites 6-8 in non-type. F. Uropods, dorsal. A-F. Ethanol-fixed materials from aquaria in London (A-D, F) and Brest (E); on petri dishes (A-B) and slides (C-F). 
POLAND (WROCLAW) • 1 q ad., BL 6.1 mm (in vial); Wroclaw Zoo; Jul. 2020; Jakub Kordas leg.; NHMW 26981 • 1 + ad., BL 6.5 mm, 2 imm., 7 juv. (in vial); same collection data as for preceding; Aug. 2020; NHMW 26982.

\section{Description of types}

All features of the diagnosis. Size of adult females 4.7-6.1 mm, males 3.7-5.4 mm. Cephalothorax comprises $32-37 \%$ body length, pleon (without telson) $48-50 \%$, telson $10-14 \%$, carapace (without rostrum) $22-34 \%$, and rostrum 3-5\%. Abdominal somites $1-5$ measure $0.6-0.9,0.7-0.9,0.7-0.9,0.6-$ 0.8 , and $0.7-0.8$ times the length of somite 6 , respectively.

CARAPACE (Fig. 4A). Carapace covers $66-93 \%$ of cephalothorax dorsally. Rostrum reaches at most to distal $2 / 3$ of artificially straight forward-oriented eyestalks (without cornea). Cervical sulcus distinct. Posterior margin leaves $0.5-2.5$ posterior thoracic somites mid-dorsally exposed. Carapace shape as in Fig. 2F, but with longer rostrum as in Fig. 4A.

EYES (Fig. 4A, C). Eyestalks and cornea dorsoventrally weakly compressed. Eyestalks with scales along basal half to $2 / 3$ of mesial margin. In dorsal view, cornea appears calotte-shaped, measuring $0.5-1.0$ times length of eyestalk (cornea not included). In lateral view the cornea appears ovoid, length 1.1-1.3 times maximum width.

Antennulae (Fig. 5B-E). Trunk extends 53-65\% of its length beyond eyes, $14-43 \%$ beyond antennal scale. Measured along dorsal midline, basal segment is 30-42\% trunk length, median segment $17-24 \%$, and terminal segment 37-51\% (Fig. 5B). Basal segment on basal half of outer face with two small, stout, barbed setae as in Fig. 2D. Dorsal apophysis with 3-4 barbed setae, a smooth, flagelliform seta, and an apically barbed, spine-like, stout seta (Fig. 5C). Lateral apophysis with four barbed setae. Median segment dorsally with large apophysis with three barbed plus a smooth, flagelliform seta, medially with large plumose seta and a flagellate spine (Fig. 5D). Terminal segment 1.1-1.2 times as long as wide. Mid-dorsal apophysis with four small barbed setae. Disto-mesial edge of terminal segment with one large flagellate spine (Fig. 5E), one large, laterally directed smooth seta, and two plumose setae in females, only one in males. The flagellate spine distally with 2-4 rounded to tooth-like tubercles (Fig. 5E). Outer antennular flagellum thicker than inner one by factor of 1.2-1.8 when measured near basis of flagella. Male lobe well setose, inserts ventrally close to terminal margin of antennular trunk, almost spherical, length is $15-24 \%$ of width of terminal segment of trunk, its width $17-22 \%$.

Antennae (Fig. 5G). The three-segmented antennal peduncle with basal segment 17-24\% peduncle length, second segment $43-55 \%$, and third segment $28-39 \%$. Sympod ventrally with stout, subrectangular, terminally rounded process (dashed line in Fig. 5G). Its outer margin ends in an apically blunt to acute projection.

MouthPARTS (Fig. 5A). Labrum as given above for $H$. smithsoniana sp. nov. Mandibular palp threesegmented. Proximal segment without setae, $10-17 \%$ length of palp. Length of median segment 2.0-2.6 times maximum width and $57-69 \%$ palp length. Inner margin of median segment with $4-5$ basally barbed setae in subbasal to terminal position. Both sexes with 8-10 smooth, shorter setae along proximal half; these setae increasing in length distally; their basal half thickened. Proximal $50-80 \%$ of outer margin all along with smooth setae, remaining distal portion with basally weakly barbed setae. Only males with small field of scales near inner basal edge on rostral face (this is below drawing plane in Fig. 5A); size and shape of scales as in Fig. 2J. Terminal segment strongly setose (most setae below drawing plane in Fig. 5A), 21-26\% palp length. Pars molaris with well-developed grinding surface in both mandibles. Pars incisiva with 3-4 teeth, digitus mobilis with 3-4 teeth, and pars centralis with three spiny teeth. Labium as described above for H. smithsoniana sp. nov. Maxillula as normal in this 


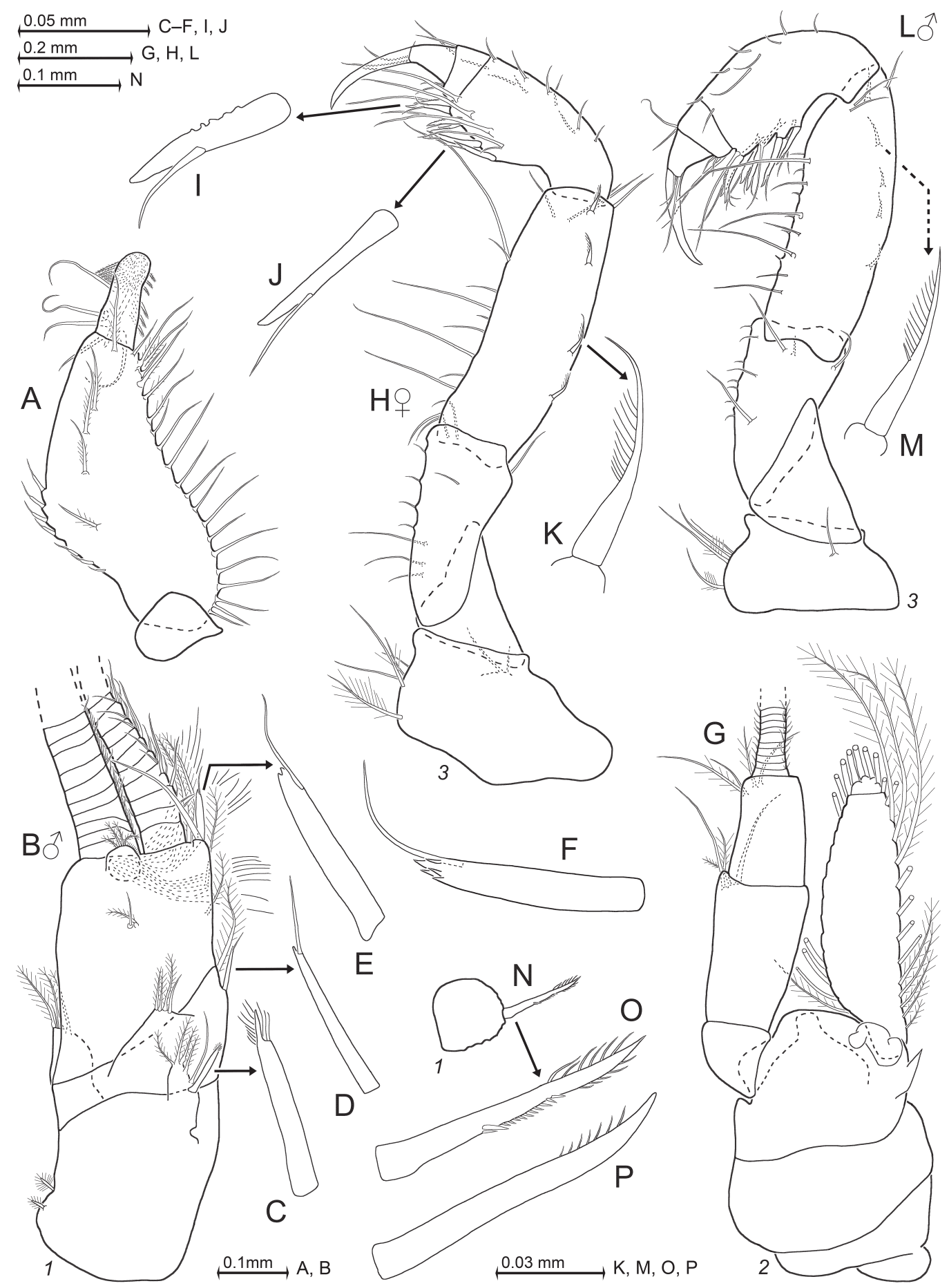

Fig. 5. Heteromysis hornimani sp. nov., paratypes from London, $q$ with body length $5.2 \mathrm{~mm}(\mathrm{~A}, \mathrm{H}-\mathrm{K}$, P: NHMW 26975), ô $4.7 \mathrm{~mm}$ (B-E, G, L-O: NHMW 26976), and non-type from Wroclaw, o $6.5 \mathrm{~mm}$ (F: NHMW 26982). A. Palp of left mandible, caudal; most setae of terminal segment are below drawing plane. B. Male antennula, dorsal. C-E. Details of male antennula (B) showing modified seta (C) and spines (D, E). F. Non-type variant of flagellate spine of terminal segment of antennula. G. Antenna with antennal gland, ventral. H. Right female thoracic sympod and endopod 3, rostral. I-K. Details of female endopod $3(\mathrm{H})$ showing modified spines (I-J) of carpus and modified seta $(\mathrm{K})$ of merus. L. Left male thoracic sympod and endopod 3, caudal. M. Detail showing modified seta of merus of male endopod 3 (L). N. Dactylus 1, setae omitted. O-P. Claw variants of dactylus $1(\mathrm{~N})$. 
genus; distal segment terminally with 11-13 smooth, in part weakly serrated spines; subterminally with 3-4 setae barbed on distal half; 2-4 pores closely adjoining outer (= most ventral) seta. Endite of maxillula terminally with three strong, distally spiny setae accompanied by $4-5$ proximally thick barbed setae; inner and outer margins with numerous less thick setae. Maxilla as normal in this genus; terminal segment of endopod with maximum width $44-76 \%$ length. Basal segment of maxillary palp with field of scales. Outer margin of exopod all along with 17-19 mostly plumose setae, two apical setae larger than remaining ones.

MaLe thoracic STERNITES (Fig. 4D). Size of mid-ventral processes increases from processes 1-4, while processes 4-8 remain subequal. Anterior 3-4 processes linguiform, terminally rounded, the posteriormost 4-5 processes triangular with acute or narrowly rounded tip. Process 1 emerges from usual mid-rostral lobe of thoracic segment 1 , remaining processes from middle to posterior third of their respective sternite.

Thoracopods (general; Figs 5H-P, 6C-G). Length of flagella as well as of basal plates increases from exopod 1 to 5 or 6 , and then decreases to exopod 8. Basal plates expanded, length 1.4-1.9 times width in both sexes. Outer margin of plate 1 ends in well-rounded edge; plates 2-8 with subrectangular, narrowly rounded to acute edge. First thoracopods with large, leaf-like, smooth epipod. Characteristic pair of setae on intersegmental joint between sympod of thoracopod 2 and corresponding sternite as in H. smithsoniana sp. nov.; no such setae in remaining thoracopods. Total length of endopod increases in series of thoracopods 1, 2, 4, 3, 5-8. Length and slenderness of ischium increase from 1 to 5 , and remain subequal among endopods 5-8. Basis of endopods 4-8 with a small, ovoid to lappet-like apophysis on rostral face below endopod, no such apophysis in endopods $1-3$. Ischium shorter than merus in endopods $1-4$ but longer than merus in endopods 5-8. Thoracic endopods with claw 3 longest; claws 1 , 4-8 roughly half length of claw 3 ; claw 2 , if any, not detected. Varying portions of claw 1 unilaterally (Fig. 5P) or bilaterally (Fig. 5O) serrated, claws 3-4 smooth (Fig. 6C-D), claws 5-8 (Fig. 6E, G) centrally to subapically serrated on inner margin. Claw 1 slightly bent, claw 4 straight, claws $3,5-8$ strongly bent. When stretched anteriorly, endopod 8 reaches to maxillula, when stretched posteriorly to end of pleonite 5 up to mid of pleonite 6 . Penes cylindrical, subbasally with 1-2 small setae.

MAXILLIPEDS. As described above for $H$. smithsoniana sp. nov., coxa of first maxilliped with small endite bearing one barbed, basally thick seta at tip. In both sexes, combined praeischium plus ischium of second maxilliped are 0.6-0.9 times length of merus, carpopropodus plus dactylus 1.0-1.2 times merus. Dactylus 2 with dense brush formed by large numbers of normal setae and 13-14 modified setae; modification as in above-quoted species.

GNaTHOPODS (third thoracic endopods; Figs 5H-M, 6C). Ischium 1.4-2.1 times as long as wide; merus 2.5-3.1 as long as wide and 1.5-2.1 length of ischium. Carpus 0.6-0.8 times length of merus, 0.9-1.2 times ischium. Claw 1.9-2.9 times length of dactylus, $47-54 \%$ carpopropodus. Series of $4-5$ unilaterally barbed, basally thickened setae along $2 / 3$ of outer face of merus; distal $2-3$ setae with most proximal barb thickened in males (Fig. 5M), normal in females (Fig. 5K). Distal half of ischium with 3-6 short whip setae on mesial margin, each whip seta on tip of a short rounded projection in both sexes. Proximal $2 / 3$ of merus with 3-6 short whip setae on mesial margin, the whip setae alternating with longer smooth setae. Carpus 3 with proximal 2-3 flagellate spines (Fig. 5I-J) in linear series, distal four spines arranged in pairs; all these spines with subapical flagellum positioned on their smooth posterior (proximal) face; 3-4 anterior (distal) spines rugged along their anterior face.

MARSuPIUM. As described above for H. smithsoniana sp. nov. Oostegite on thoracopod 7 near basis with 6-9 microserrated setae, larger oostegite on thoracopod 8 with 3-5 such setae. Ventral and rostral portions of outer face of only larger oostegite with total of 14-19 flagelliform setae which are shorter than barbed setae present in dense series along lower margin. Thoracopod 6 with rudimentary oostegite represented by small, rounded lobe terminally with three setae which are microserrated on distal half. 
Pleopods (Fig. 7). Size increases in series of pleopods 1, 3, 4, 5, 2 in females, and 1, 5, 2, 3, 4 in males. For presence and numbers of spines on pleopods 2-4, see 'Diagnosis' above. All setae of pleopods 1-5 plumose or barbed in both sexes (not counting flagellate spines). Most specimens of both sexes, including holotype, show peculiar perpendicular orientation of only second pleopod (as in Fig. 8C).

TAIL FAn (Figs 4F, 6H-I). Scutellum paracaudale triangular, tip acute or narrowly to well rounded. Exopod of uropods extends by 4-25\% its length beyond endopod, $25-47 \%$ beyond telson; endopod $28-$ $43 \%$ its length beyond telson. Diameter of fluorite statoliths is $80-106 \mu \mathrm{m}(\mathrm{n}=10$ statoliths from five specimens). Statoliths discoidal with shallow fundus and distinct tegmen. Statolith formula $2+3+(0-1)$

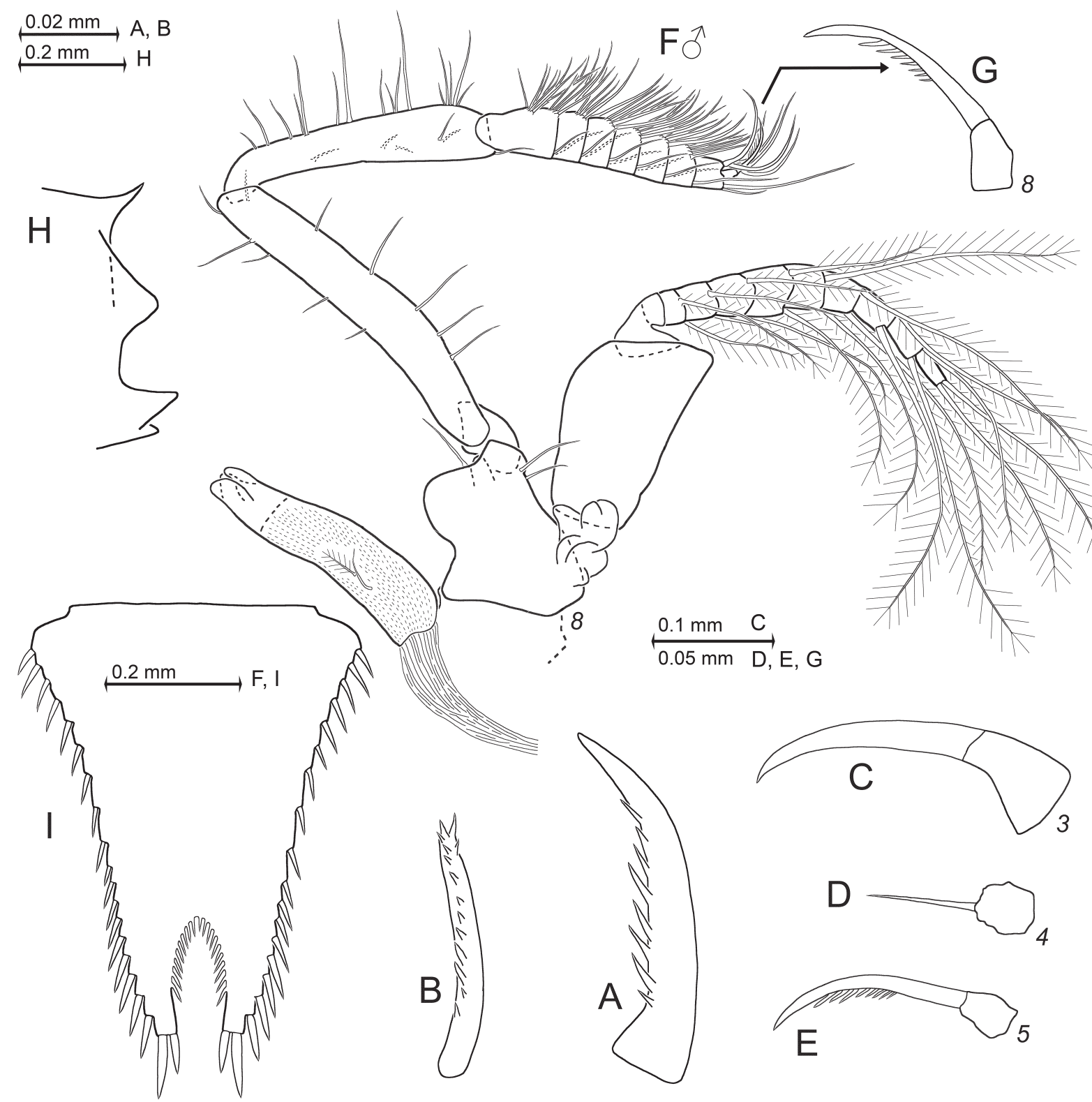

Fig. 6. Heteromysis hornimani sp. nov., paratypes, $q$ with body length $5.2 \mathrm{~mm}(\mathrm{~A}-\mathrm{C}, \mathrm{H}-\mathrm{I}$ : NHMW 26975) and $7.7 \mathrm{~mm}$ (D-G: NHMW 26976). A-B. Modified spines from dorsolateral infoldings (A) and lateralia (B) of foregut. C-E. Series of dactylus 3-5, with claws, setae omitted. F. Thoracopod 8 with penis, rostral. G. Detail of endopod 8 (F) showing dactylus with nail. H. Scutellum paracaudale, lateral. I. Telson. 
$+(5-10)+(6-10)=19-22$. Telson length 1.2-1.5 times its maximum width and 0.9-1.2 times length of sixth pleonite. Spine length on lateral margins of telson decreases in continuous series from basis to half-length and from there increases up to tip. For further details of telson, see 'Diagnosis' above.

FOREGUT (Fig. 6A-B) essentially as described above for $H$. smithsoniana sp. nov. Caudal portion of lateralia in H. hornimani sp. nov. with group of 3-5 small, apically pronged spines serrated along distal $3 / 4$ of shaft (Fig. 6B). Dorsolateral infoldings with group of three longer, apically smooth, subbasally to subapically serrated spines (Fig. 6A). Gut contents of three specimens examined were mostly unidentifiable material and mineral particles, a few fragments of filiform algae, no arthropod remains.
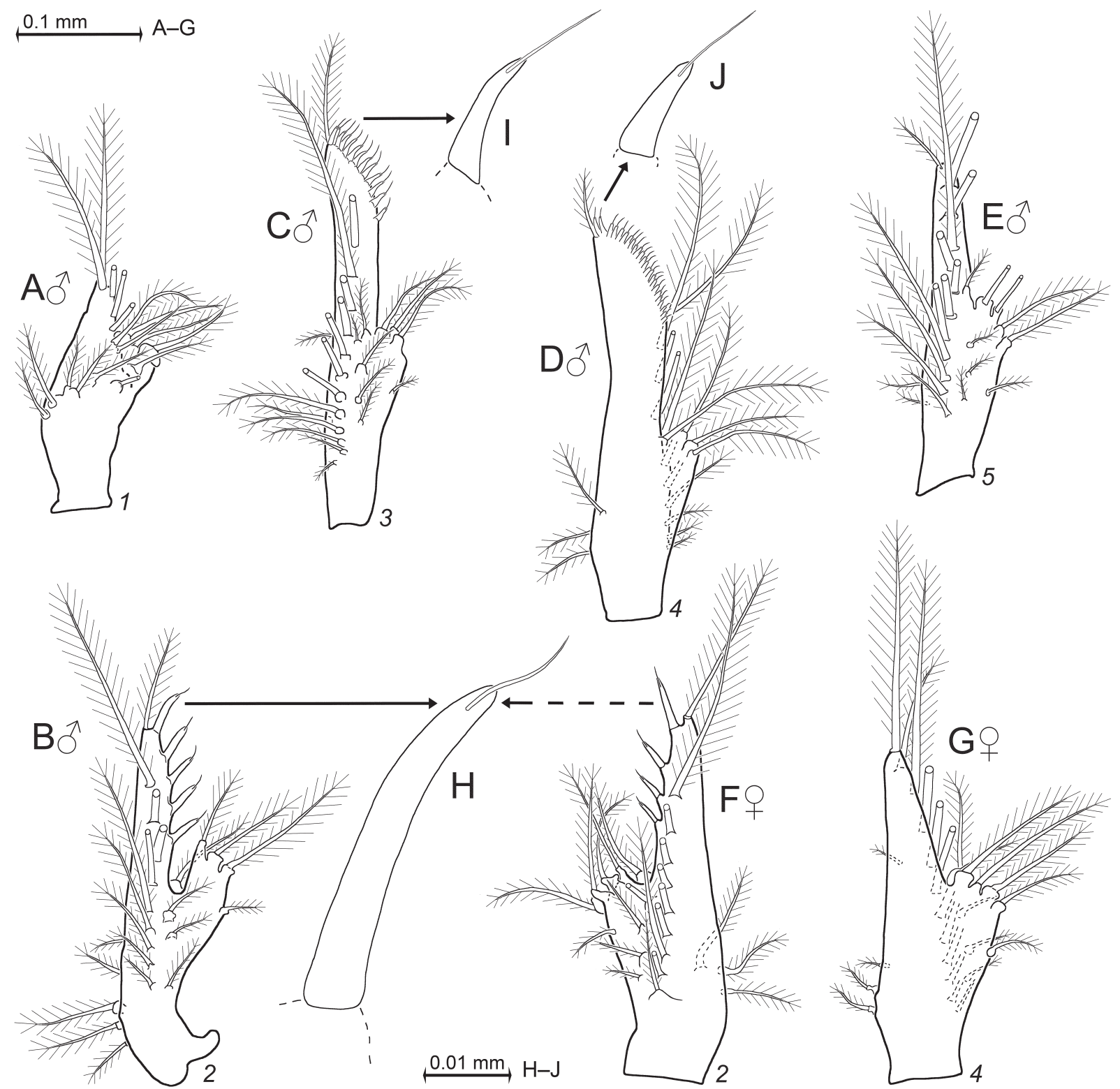

Fig. 7. Heteromysis hornimani sp. nov., paratypes from London, $\widehat{\jmath}$ with body length $4.7 \mathrm{~mm}$ (A-E, H-J: NHMW 26976), $95.2 \mathrm{~mm}$ (G: NHMW 26975), and non-type from Brest, $q 4.3 \mathrm{~mm}$ (F: NHMW 26979). A-E. Series of male pleopods 1-5; rostral (A-C, E) or caudal (D) face. F. Female pleopod 2, rostral. G. Female pleopod 4, caudal. H-J. Series of flagellate spines of male pleopods 2-4 (B-D). 

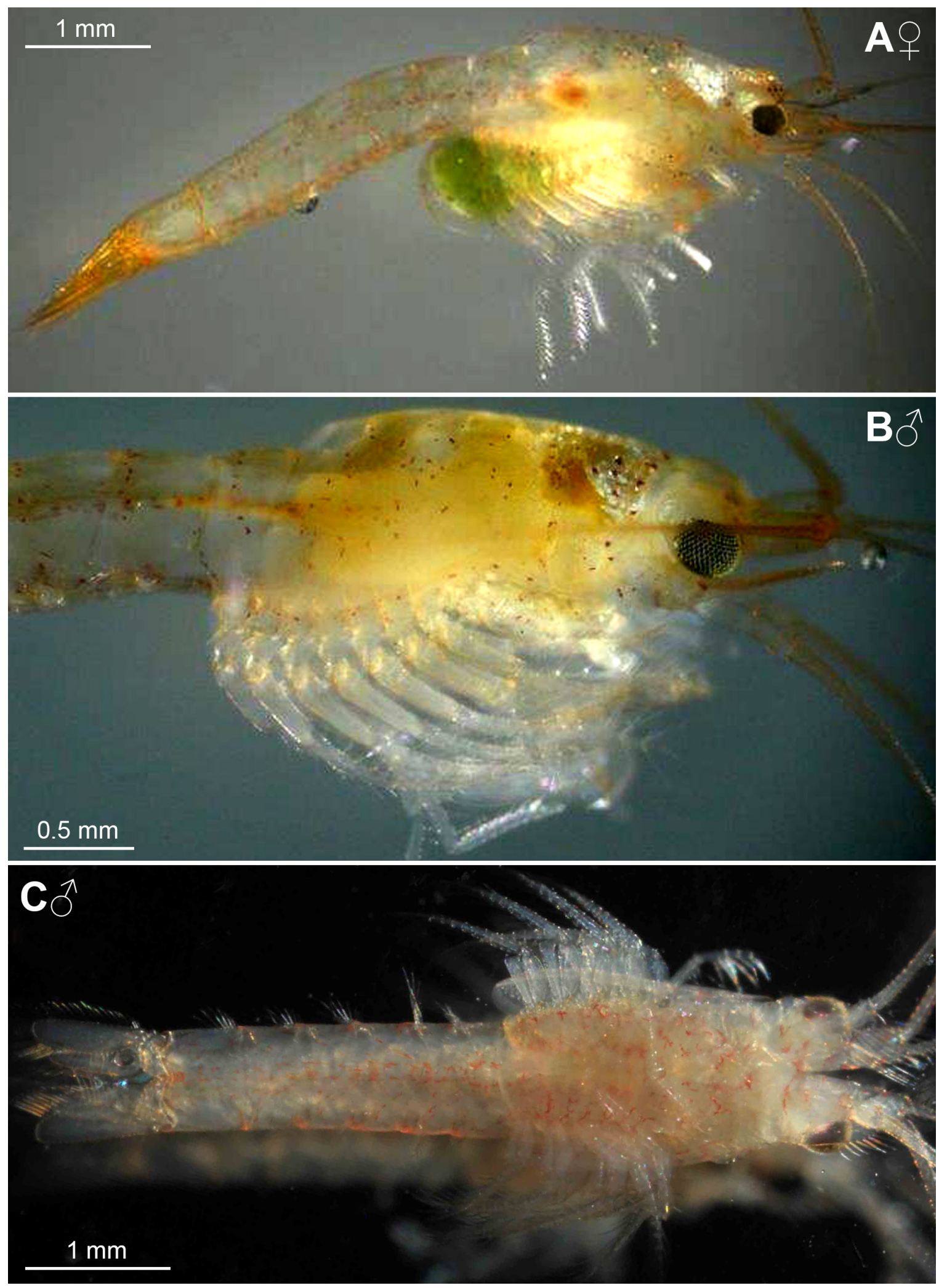

Fig. 8. Heteromysis hornimani sp. nov., non-types from aquaria in Brest (A-B) and London (C). A. 9 , lateral, note green eggs in marsupium. B. Anterior half of adult $\hat{\partial}$, lateral. C. Adult $\hat{\partial}$, ventral, note perpendicular orientation of pleopod 2. A-C. Random specimens from sample \# 6 (A-B) and \# 3 (C); laboratory photos of live specimens by Dominique Barthelmy (A-B) and Jamie Craggs (C). 


\section{Colour}

РнотоS FRom Brest (Fig. 8A-B). General appearance transparent with yellow to orange-brown portions, rendering contents of foregut and alimentary canal visible. Small orange to brown chromatophore spots scattered over the thorax, with greatest density above the foregut. The reflecting spots above the foregut may be due to small oil globules (usually disappearing in ethanol-preserved material). Eggs are green, as often found in species of Heteromysis.

Рнотo From London (Fig. 8C). Compared with the figures from Brest, the figure from London shows an essentially similar density of chromatophores, but more 'expanded' ones, giving the animal a more intensive orange to red tinge. The differences between Fig. 8A-B and Fig. 8C are well within the range of normal colour change in species of the family Mysidae.

\section{Distribution}

The species is so far known only from tanks in the 'Horniman Museum Aquarium London' (UK), the 'Oceanopolis Aquarium' in Brest (France), the 'Aquarium de Paris' (France), and the 'Zoo Wroclaw' (Poland). The data from Paris suggest a Caribbean origin (Table 1: \# 4).

\section{Eggs and larvae}

Material from London. Two females with empty brood pouch, while another female with $5.3 \mathrm{~mm}$ body length carried three postnauplioid larvae at substage P2 with $1.28-1.35 \mathrm{~mm}$ length, one female $6.1 \mathrm{~mm}$ carried three P2 with $1.23-1.35 \mathrm{~mm}$. A crude estimate suggests that the larvae attain $1 / 4$ parent length shortly before the moult to the free-living juvenile stage.

Material from Wroclaw. Female with $6.1 \mathrm{~mm}$ body length carried one nauplioid larva at substage N1 (0.92 mm length); female $6.5 \mathrm{~mm}$ carried two eggs with diameter $0.49 \mathrm{~mm}$ and eight N1-larvae with $0.87-1.11 \mathrm{~mm}$. The co-occurrence of eggs and N1-larvae in the same brood pouch is rarely found. This indicates that the embryos were asynchronously hatching from the egg membrane shortly before and during fixation. The observed strong size differences between the hatchlings are clearly due to the obligatory stretching of the abdomen during and shortly after hatching. Adoption of eggs or larvae from other mothers appears unlikely in this case: in experiments of Wittmann (1978) with mysids from other subfamilies, the breeding females did not introduce offered eggs (embryos) into their brood pouch, but a few mothers did so with up to two nauplioid larvae, never with more. The here studied N1-larvae show a smooth cuticle all around.

\section{Heteromysis (Olivemysis) waikikensis sp. nov. urn:lsid:zoobank.org:act:C1DBD087-302A-47F9-BD48-7924DB1BBFBE} Figs 9-11

\section{Diagnosis}

Rostrum (Fig. 9A) forms a triangle with an acute or narrowly rounded tip, 50-65\% length of terminal segment of antennular trunk. Cornea (Fig. 9A-C) occupies distal 30-40\% eye surface. Eyestalks with smooth surface except for a prominent tooth on disto-mesial edge. Antennular trunk (Fig. 10A-D) with two large, subapically flagellate spines in addition to normal setae, namely one spine on disto-mesial edge of median segment, and a broader one on disto-mesial edge of terminal segment. Distal $8-12 \%$ of the latter spine rugged on its obliquely truncate margin. Dorsal apophysis of basal segment with one strong, apically rugged, non-flagellate spine. Antennal scale (Fig. 10E) and antennal peduncle ending at about same height; scale length is 2.9-3.3 times maximum width; scale with small apical segment separated by a transverse suture. Male thoracic sternites 1-8 each with one median, ventral process (Fig. 9D). Females with smooth thoracic sternites. Both sexes with flagellum of thoracic exopod 1 showing eight 
segments, exopods $2-8$ with nine segments (Fig. 11A). Carpopropodus of thoracic endopods $1-8$ with 2 , 2, 2, 4, 7, 7, 7, and 7 segments, respectively (Figs 10I, L; 11A). Thoracic endopod 3 (Fig. 10I, L) without tooth-like extension on disto-mesial edge of merus; carpopropodus length 2.2-2.7 times maximum width in both sexes. Distal $37-50 \%$ of carpus 3 with total of $6-9$ flagellate spines along inner margin; propodus without paradactylary setae or spines. Thoracopods 7-8 with large oostegites, thoracopod 6 with rudimentary oostegite. Penes (Fig. 11A) cylindrical, 80-89\% length of ischium 8 . Penes with setae on basal half; penes apically blunt, ending in four lobes. Pleopods (Fig. 11C-L) rudimentary, unsegmented, with residual differentiation of endopod (pseudobranchial lobe). Female pleopods 1, 3-5 and male pleopods 1, 5 with normal setae only, no spines. Pleopod 2 of both sexes with smooth spine (stylet) on apex and 5-6 minute flagellate spines in series along outer (= lateral) margin of elongate distal portion. Pleopods 3-4 dimorphic. Male pleopod 3 with 2-7 large flagellate spines (numbers include bilateral variability), densely set along obliquely truncate, terminal margin; male pleopod 4 with dense series of 11-12 (intermediate in size between the spines of pleopods 2 and 3) flagellate spines in analogous position. Exopod of uropods (Fig. 9E) 1.2-1.4 times length of endopod. Endopod with only one small spine near statocyst; distal spine-free portion is $67-82 \%$ length of endopod. Each lateral margin of telson (Fig. 9F) with 8-10 spines along distal 48-52\%, proximal portion smooth. U-shaped apical cleft penetrates $25-30 \%$ length of telson; proximal $87-90 \%$ of cleft with $25-32$ laminae. Distolateral lobes each with two spines on narrowly truncate apex. The outer apical spines are $15-17 \%$ length of telson; inner apical spines are $0.3-0.5$ times length of outer ones.

\section{Etymology}

The species name is a feminine adjective formed by the addition of location suffix, related to the 'Waikiki Aquarium' (Honolulu, Hawaii) where the new species was detected.

\section{Material examined}

\section{Holotype}

HAWAII • $\widehat{O}$ ad., BL 3.9 mm (in vial); Honolulu, Waikiki Beach, Waikiki Aquarium; Jan. 2020; Gwen Lentes leg.; NHMW 26965.

\section{Paratypes}

HAWAII 1 1 ad., BL $4.2 \mathrm{~mm}$ (on slides); same collection data as for holotype; NHMW 26966 • $1 \delta$ ad., BL $4.2 \mathrm{~mm}$ (on slides); same collection data as for holotype; NHMW 26967.

\section{Description}

All features of diagnosis. General appearance small, robust (Fig. 9A). For body size see above 'Material examined'. Cephalothorax comprises $28-34 \%$ of body length, pleon (without telson) $54-67 \%$, and carapace (without rostrum) 20-31\%. Abdominal somites $1-5$ measure $0.7-1.0,0.6-1.0,0.7-0.9,0.7-$ 0.9 , and $0.6-1.0$ times the length of somite 6 , respectively.

CARAPACE (Fig. 9B). Carapace covers $70-81 \%$ of cephalothorax dorsally. Rostrum reaches at most to distal $2 / 3$ of artificially straight forward-oriented eyestalks (without cornea). Cervical sulcus short but strong. Cervical pore group consists of 11-15 pores with about $1 \mu \mathrm{m}$ diameter in a roughly U-shaped arrangement. Posterior margin leaving 1-2 posterior thoracic somites mid-dorsally exposed.

EYES (Fig. 9A-C). Eyestalks and cornea dorsoventrally compressed (Fig. 9A). Eyestalks without scales. In dorsal view (Fig. 9B-C) the cornea appears calotte-shaped, measuring 0.8-1.0 times length of eyestalk (cornea not included). In lateral view (Fig. 9A) the cornea appears ovoid with flattened ventral margin, length 1.5 times maximum width.

Antennulae (Fig. 10A-D). Trunk extends 40-58\% its length beyond eyes, 19-40\% beyond antennal scale. Measured without apophysis along dorsal midline, basal segment is $32-47 \%$ trunk length, median 

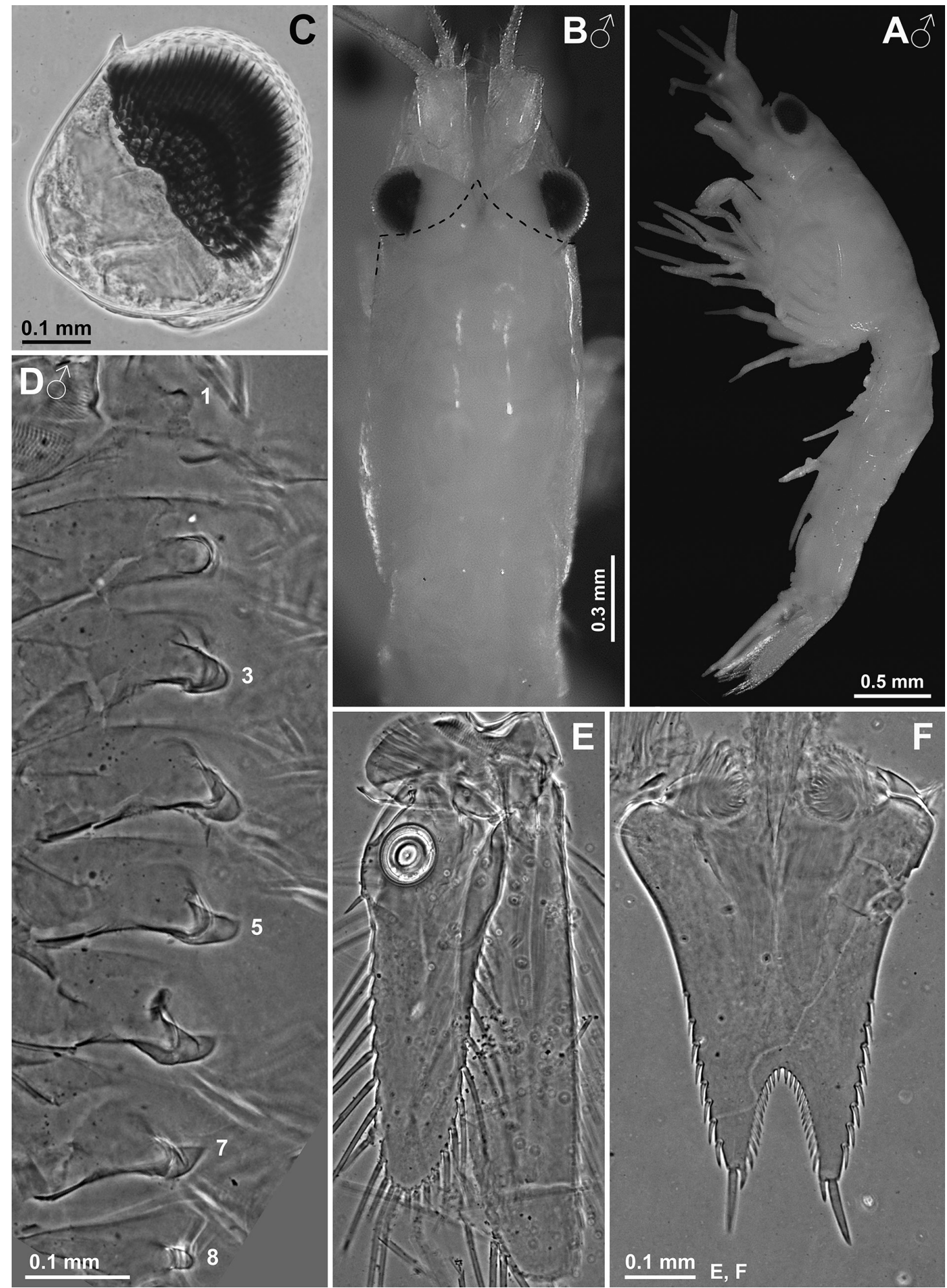

Fig. 9. Heteromysis waikikensis sp. nov., holotype, ô with body length $3.9 \mathrm{~mm}$ (A-B: NHMW 26965),

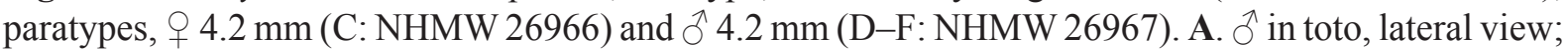
figure artificially separated from background. B. Cephalothorax, dorsal view; dashed line illustrates the anterior contour of carapace. C. Right eye, dorsal view; object somewhat compressed by cover glass pressure. D. Thoracic sternites 1-8 expanded on slide, ventral. E. Uropods, ventral. F. Telson. A-F. Ethanol-fixed materials on petri dishes (A-B) and slides (C-F). 
segment $13-16 \%$, and terminal segment $48-54 \%$. Basal segment on basal half of outer face with two small, stout, barbed setae as in Fig. 2D. Its dorsal apophysis with 4-5 barbed setae, one flagelliform seta and one strong, apically rugged, non-flagellate spine (Fig. 10B). Lateral apophysis with $4-5$ barbed plus one smooth seta. Median segment dorsally with large apophysis bearing three barbed plus one flagelliform seta. Terminal segment 1.2-1.5 times as long as wide. Mid-dorsal apophysis with 4-5 barbed setae. Outer antennular flagellum thicker than inner one by a factor of 1.3-1.5 when measured near basis of flagella. Male lobe setose, inserts ventrally close to terminal margin of antennular trunk, length is $18-26 \%$ width of terminal segment of trunk, its width $15-28 \%$.

Antennae (Fig. 10E). Three-segmented antennal peduncle with basal segment 16-22\% peduncle length, second $44-49 \%$, and third 33-36\%. Sympod with tongue-like, terminally broad process on ventral face.

MouthPARTS (Fig. 10F-H). Labrum as given above for H. smithsoniana sp. nov. Mandibular palp (Fig. 10F-H) three-segmented. Its proximal segment without setae, $11-15 \%$ length of palp. Length of median segment 2.3-2.6 times its maximum width and 60-67\% palp length. Inner margin of median segment with 4-2 smooth and sparsely barbed setae in subapical to subbasal position, and with 6-8 short setae along proximal half in both sexes. These setae increasing in length distally; their basal half thickened; only in males with unilateral series of stiff barbs; distal half smooth (Figs 10G vs 10H). Outer margin of median segment all along with basally barbed setae. Only males with small field of scales near inner basal edge on rostral face; size and shape of scales as in H. smithsoniana sp. nov. (Fig. 2J). Terminal segment densely setose, $18-27 \%$ palp length. Pars molaris with well-developed grinding surface in both mandibles. Pars incisiva with four teeth, digitus mobilis with three teeth, and pars centralis with 2-3 spiny teeth. Labium as described above for $H$. smithsoniana sp. nov. Maxillula as normal in this genus; distal segment terminally with 12-14 smooth, in part weakly serrated spines; subterminally with three setae barbed on distal half; two pores closely adjoining outer (= most ventral) seta. Endite of maxillula terminally with three strong, distally spiny setae accompanied by four proximally thick barbed setae; inner and outer margins with numerous less thick setae. Maxilla as normal in this genus; terminal segment of endopod with maximum width 54-64\% length. Outer margin of exopod all along with 15-17 mostly plumose setae, two apical setae larger than remaining ones.

MALE THORACiC STERNites. Size increases from sternal processes 1-3, remains subequal from 3 to 6 , and decreases from 6 to 8 (Fig. 9D).

THORACOPODs (general; Figs 10I-M, 11A-B). Length of flagella as well as of basal plates increases from exopod 1 to 6 and decreases from exopod 6 to 8. Basal plates (Fig. 11A) expanded, length 1.6-2.1 times width in both sexes. Outer edge of plates angular, tip pointed or narrowly rounded. First thoracopods with large, leaf-like, smooth epipod. Basis of endopods 4-8 with small, lappet-like apophysis on rostral face below endopod (below drawing plane, therefore visualized by dashed lines in Fig. 11A). Total length of endopods increases in series of thoracopods 1, 2, 4, 3, 5-8. Ischium becomes longer and more slender from endopods $1-6$, while both features remain subequal among endopods $6-8$. Ischium shorter than merus in endopods 1-4 (Fig. 10I, L), but longer than merus in endopods 5-8 (Fig. 11A). Thoracic endopods with claw 3 longest, claw 1 about half length of claw 3 , claws $4-8$ measure $1 / 3$ to $1 / 2$ of claw 3 ; claw 2, if any, not detected. Claws 3-4 smooth (Fig. 10I, L), claws 5-8 subapically unilaterally serrated (Fig. 11B), claw 1 uni- or bilaterally serrated (as in Fig. 5O-P). Claw 4 straight, claws 1, 3 weakly bent (Fig. 10I, L), claws 5-8 strongly bent (Fig. 11B). When stretched anteriorly, endopod 8 reaches to labrum, when stretched posteriorly to end of pleonite 5. Penes with 2-3 barbed plus one smooth seta on proximal half (Fig. 11A). 


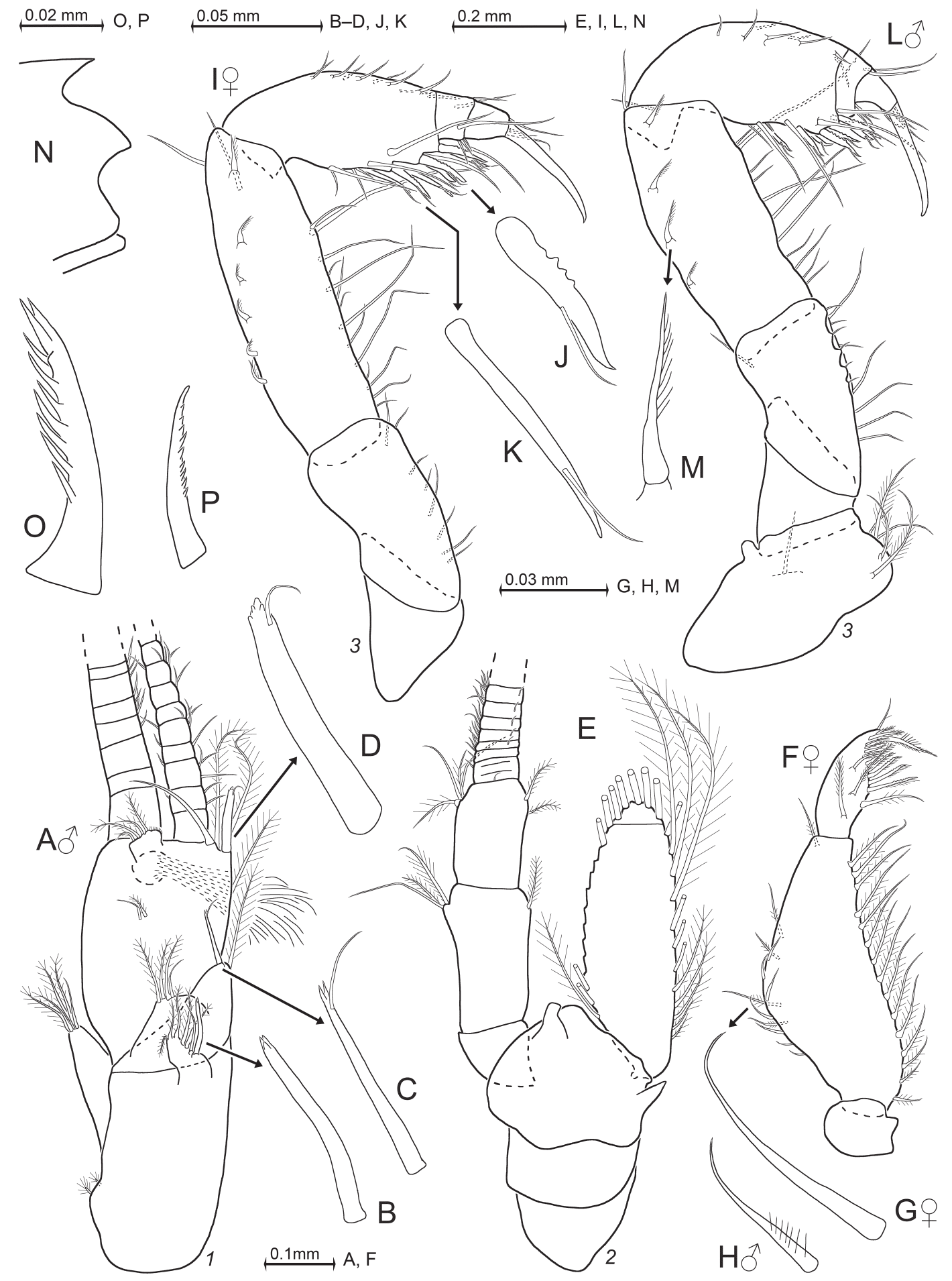

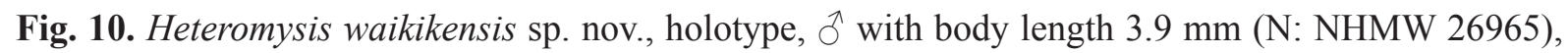
paratypes, ô $4.2 \mathrm{~mm}$ (A-E, L-M, O-P: NHMW 26967) and $\$ 4.2 \mathrm{~mm}$ (F-K: NHMW 26966). A. Male antennula, dorsal. B-D. Details of male antennula (A) showing modified spines. E. Antenna with antennal gland, dorsal. F. Palp of left mandible, caudal. G-H. Setae from outer margin of median segment of mandibular palp in $\Phi(\mathrm{G})$ versus $\hat{\partial}(\mathrm{H})$. I. Left female thoracic endopod 3, rostral. J-K. Details of female endopod (I) 3 showing modified spines of carpus. L. Left male thoracic sympod and endopod 3, rostral. M. Detail of male endopod 3 (L) showing modified seta of merus. N. Scutellum paracaudale, lateral. O-P. Modified spines from dorsolateral infoldings $(\mathrm{O})$ and lateralia $(\mathrm{P})$ of foregut. 


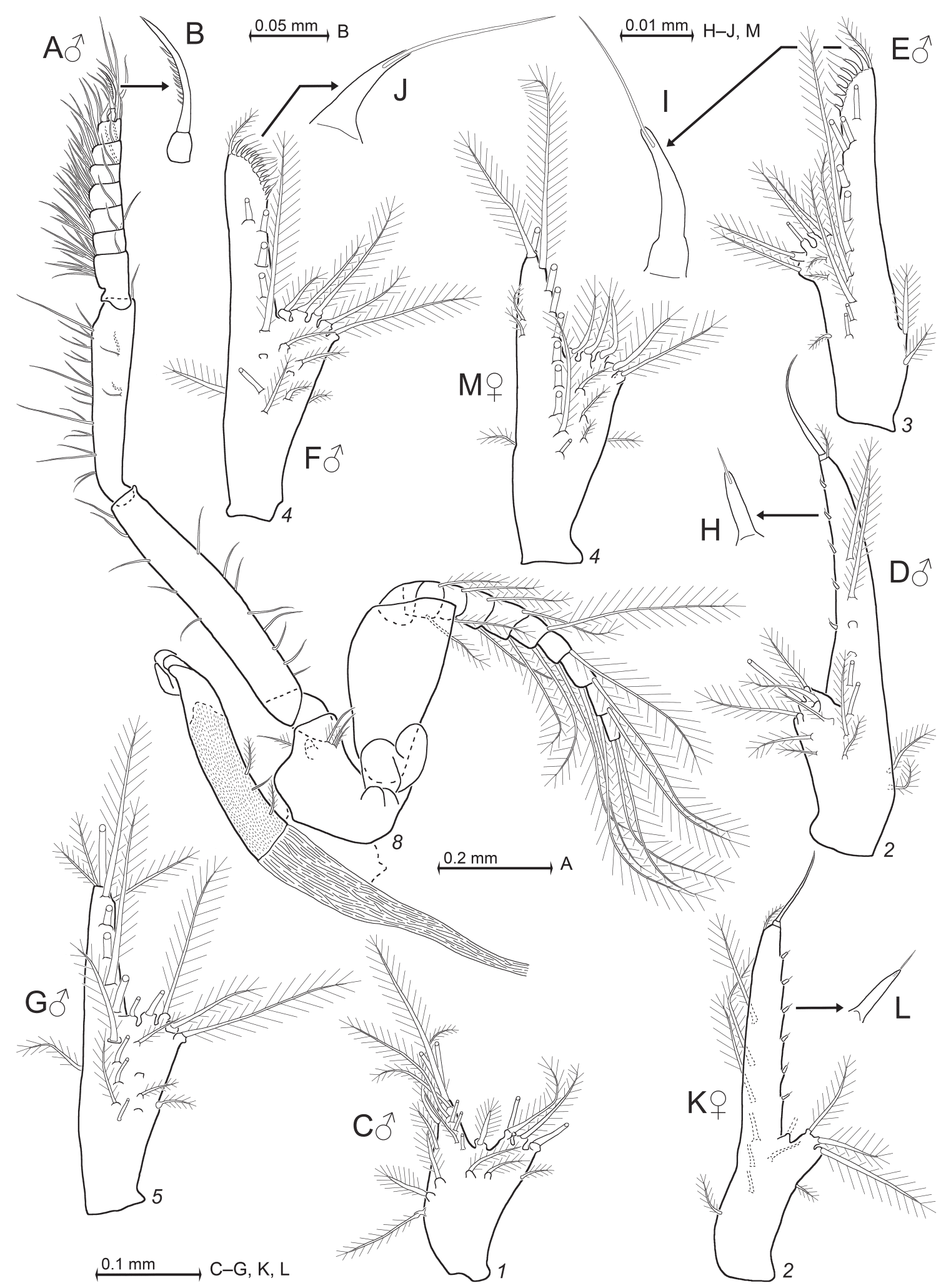

Fig. 11. Heteromysis waikikensis sp. nov., paratypes, o $4.2 \mathrm{~mm}$ (A-J: NHMW 26967) and $q 4.2 \mathrm{~mm}$ (K-M: NHMW 26966). A. Thoracopod 8 with penis, rostral view. B. Detail of thoracic endopod 8 (A) showing dactylus with nail. C-G. Male pleopods $1-5$, represented by left $(C, F-G)$ and right (D-E) ones; caudal. H-J. Flagellate spines of male pleopods 2-4 (D-F). K. Right female pleopod 2, rostral. L. Detail of female pleopod 2 (K) showing flagellate spine. M. Left female pleopod 4, caudal. 
MAXILLIPEDS. First maxilliped as described above for $H$. smithsoniana sp. nov. Basis of second maxilliped with large, distinctly medially projecting endite. In both sexes, combined praeischium plus ischium are 0.8-0.9 times length of merus, carpopropodus plus dactylus 0.9-1.1 times merus. Dactylus very large, with dense brush formed by large numbers of normal setae and 11-13 modified setae, the latter apically bent, bearing two symmetrical series of denticles (stiff barbs) on either side in subbasal to median portions.

GNATHOPODs (thoracic endopods 3; Fig. 10I-M). Ischium 1.2-2.2 times as long as wide; merus 2.0-3.6 as long as wide and 1.5-2.2 length of ischium. Carpus 0.5-0.8 times length of merus, 0.9-1.1 times ischium. Claw 2.6-3.9 times dactylus length, and 45-50\% carpopropodus. Both sexes with series of basally thickened, unilaterally barbed setae (Fig. 10M) on distal $71-80 \%$ of outer face of merus. These setae with series of normal barbs (cilia) along median to subapical portions, basal portions thickened. Distal half of ischium with 4-5 short whip setae on mesial margin, each whip seta on tip of a short rounded projection in males, no such projections in the only female available in the present material. Proximal $2 / 3$ of merus with $3-6$ short whip setae on mesial margin, the whip setae alternating with longer smooth setae; these setae not implanted on projections. Carpus 3 with proximal 1-2 flagellate spines (Fig. 10K), in case of two spines one behind the other; distal spines arranged in 2-4 pairs (one pair after the other); spines of only the most distal 2-3 pairs with rugged anterior margins (Fig. 10J).

MARSuPIUM. Oostegites 1-2 (derivates of thoracopods 7-8) without setae on upper (dorsal) margins. Lower margins from subbasal region up to the rounded tip bearing series of setae, most of which barbed by fine cilia along subbasal to median portions. Oostegite 1 near basis with $6-9$ setae, oostegite 2 with 5-7 setae which are sparsely microserrated along distal half. Ventral and rostral portions of outer face of only second oostegite with total of 9-11 whip setae, but shorter than barbed setae. Thoracopod 6 with rudimentary oostegite represented by small, rounded lobe terminally with four setae which are microserrated on their distal half.

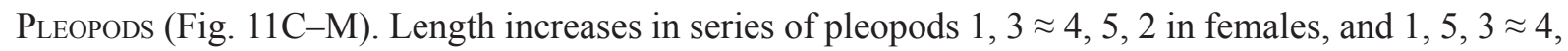
2 in males. For presence and numbers of spines on pleopods 2-4, see 'Diagnosis' above. In Fig. 11D, the flagellate spines are perspectively shortened by slightly oblique orientation of pleopod. All setae of pleopods $1-5$ plumose or barbed in both sexes.

TAIL FAN (Figs 9E-F, 10N). Scutellum paracaudale triangular, tip acute or narrowly rounded (Fig. 10N). Exopod of uropods extends by $14-21 \%$ its length beyond endopod, or $40-49 \%$ beyond telson. Endopod reaches with $24-29 \%$ its length beyond telson. Statoliths mineralised with fluorite, diameter $70-110 \mu \mathrm{m}$, thickness 40-45 $\mu \mathrm{m}(\mathrm{n}=4)$. Statoliths discoidal with shallow fundus and distinct tegmen. Statolith formula $2+(2-3)+(1-2)+(5-9)+(4-5)=15-20$. Telson length 1.3-1.4 times its maximum width or 0.9-1.0 times length of endopod of uropod, 0.8-0.9 times exopod of uropod, and 1.1-1.4 times last abdominal somite, respectively. For further details of telson, see 'Diagnosis' above.

Foregut (Fig. 10O-P) essentially as described above for $H$. smithsoniana sp. nov. (Fig. 3A-B). However, certain modified spines more similar to those (Fig. 6A-B) of $H$. hornimani sp. nov., namely 1-2 large, apically pronged, serrated spines (Fig. 10O) on dorsolateral infoldings, and group of 4-5 smaller, centrally serrated spines (Fig. 10P) on posterior part of lateralia. Gut contents of two dissected specimens were mostly unidentifiable material, few mineral particles and crustacean fragments.

\section{Distribution}

The species is so far known only from an aquarium tank of the 'Waikiki Aquarium', Honolulu, Hawaii. Origin most likely in coastal marine waters of the Central Pacific (Table 1). 


\section{Heteromysis (Olivemysis) sixi sp. nov \\ urn:Isid:zoobank.org:act:A5C4DF45-0137-4A0F-AAB5-4C71E081755C}

Figs $12-13$

\section{Diagnosis}

Only male holotype known (ranges of meristic characters are here indicating differences between left and right side of the body). Rostrum (Fig. 12A) triangular with narrowly rounded tip, $2 / 3$ length of terminal segment of antennular trunk. Cornea (Fig. 12A-B) occupies distal third of eye surface. Eyestalks with smooth surface except for prominent tooth on disto-mesial edge. Antennular trunk (Fig. 12C-E) with blade-like, subapically flagellate spine in addition to setae on disto-mesial edge. Distal $16-24 \%$ of this spine with rugged, obliquely truncate margin. Antennal scale (Fig. 12A) undivided, extending shortly beyond antennal peduncle; scale length 2.4-3.0 times maximum width. Thoracic sternites (Fig. 12H) with short median processes. Flagellum of thoracic exopod 1 with 8 segments, exopods $2-8$ with nine segments (Fig. 13A). Carpopropodus of thoracic endopods 1-8 with 2, 2, 2, 4, 6, 6, 6, and 6 segments, respectively. Thoracic endopod 3 (Fig. 12I-M) without tooth-like extension on disto-mesial edge of merus; carpopropodus length 2.5 times maximum width. Distal $44-64 \%$ of male carpus 3 with total of six flagellate spines along inner margin; propodus without paradactylary setae. Penes (Fig. 13A) cylindrical, apically blunt, ending in five blunt lobes; size $3 / 4$ length of ischium 8 ; small setae present at half length of penis. Pleopods (Fig. 13B-G) rudimentary, unsegmented, with residual differentiation of endopod (pseudobranchial lobe). Male pleopods 1, 3-5 normal, without spines. Pleopod 2 with large smooth spine (stylet) on apex and three minute flagellate spines in series along distal half of outer margin of elongate distal portion. Exopod of uropods (Fig. 13H) 1.2-1.3 times length of endopod; endopod with only one small spine near statocyst; distal spine-free portion $2 / 3$ length of endopod. Each lateral margin of telson (Fig. 13I) with six spines on distal half, proximal portion smooth. U-shaped apical cleft penetrates $1 / 3$ telson length; proximal $5 / 6$ of cleft with 26 laminae. Disto-lateral lobes each with two spines on narrowly truncate apex. The outer apical spines are 16-18\% telson length; inner apical spines are $0.2-0.3$ times length of outer ones.

\section{Etymology}

The species name is a masculine noun in genitive singular, dedicated on the occasion of his retirement to Franz Six, citizen scientist and 55 year-long promoter of the 'Haus des Meeres', a public marine aquarium in Vienna.

\section{Material examined}

\section{Holotype}

HAWAII • $\widehat{\text { â }}$ ad., BL $3.0 \mathrm{~mm}$ (on slides); Honolulu, Waikiki Beach, Waikiki Aquarium; Jan. 2020; Gwen Lentes leg.; NHMW 26964.

\section{Description}

All features of the diagnosis. Cephalothorax comprises $30 \%$ of body length, pleon (without telson) $55 \%$, and carapace (without rostrum) 32\%. Abdominal somites 1-5 measure 1.0, 0.9, 0.8, 0.9, and 0.9 times the length of somite 6 , respectively.

CARAPACE (Fig. 12A). Carapace covers $75 \%$ of cephalothorax dorsally. Rostrum reaches to proximal third of artificially straight forward-oriented eyestalks (without cornea). Cervical sulcus short but strong. Posterior margin leaving 1-2 posterior thoracic somites mid-dorsally exposed.

Eyes (Fig. 12A-B). Eyestalks and cornea dorsoventrally compressed (Fig. 12B). Eyestalks without scales. In dorsal view (Fig. 12A) the cornea appears calotte-shaped, measuring $4 / 5$ length of eyestalk 

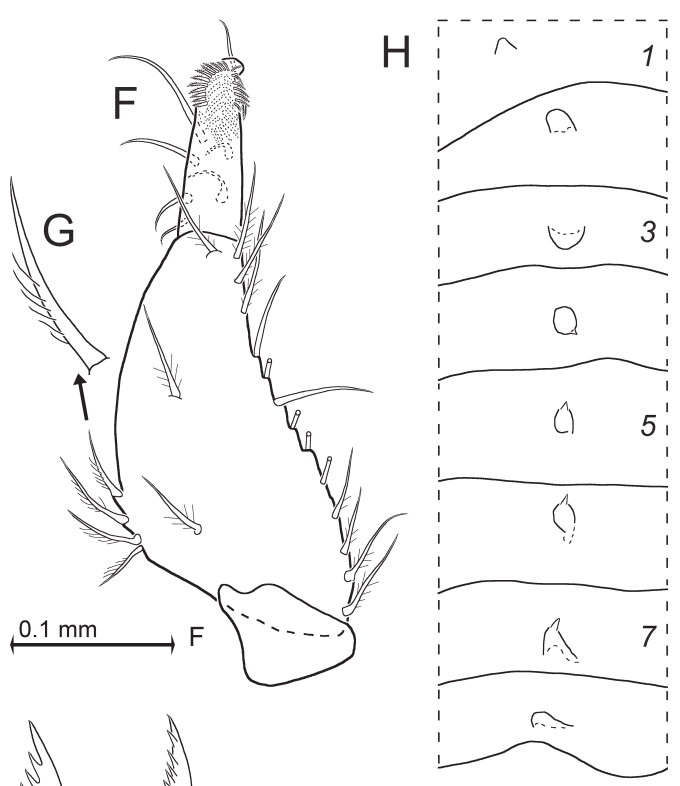

$0.03 \mathrm{~mm}$ $D, E, G, J-L$
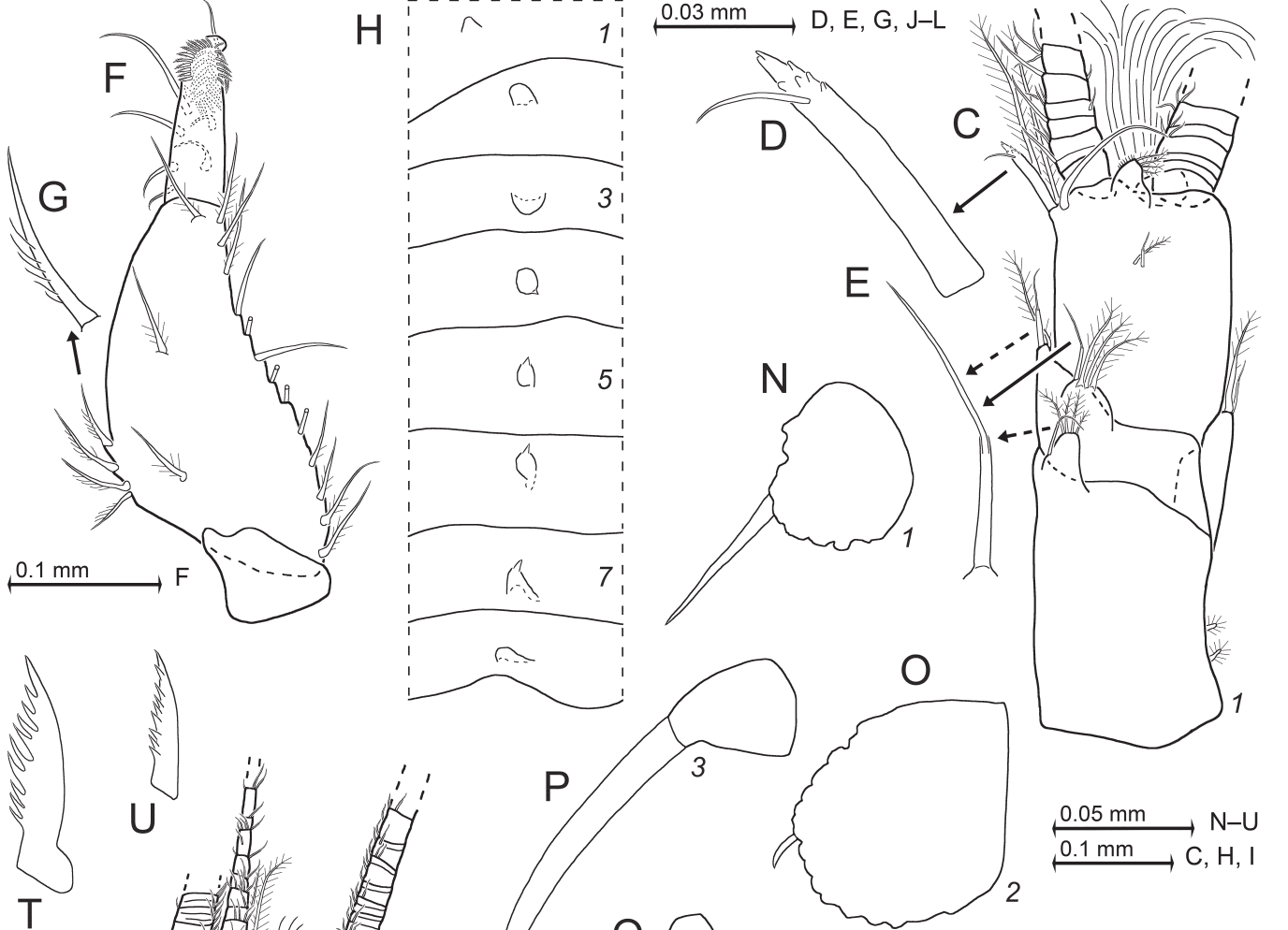

E

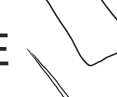

$S \underbrace{2}$
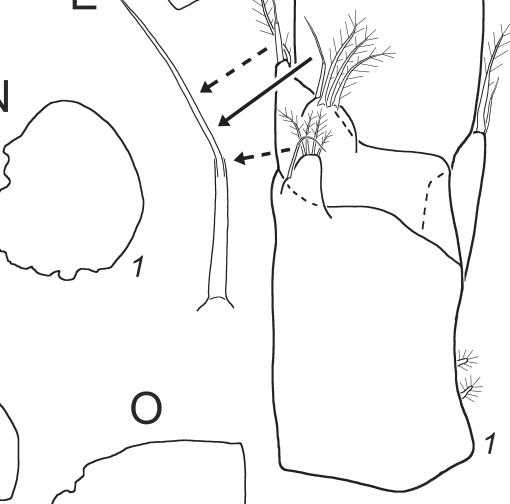

Fig. 12. Heteromysis sixi sp. nov., holotype, ô with body length $3.0 \mathrm{~mm}$ (NHMW 26964). A. Anterior body region, dorsal view; right antennula, left antenna, and setae of antennal scale omitted. B. Detail of panel (A) showing right eye in lateral view. C. Right antennula, dorsal. D-E. Details of antennula (C) showing flagellate spine (D) and an example for whip setae (E) found here and on other appendages. F. Palp of left mandible, caudal. G. Detail of panel (F) showing barbed seta from outer margin of median segment. H. Thoracic sternites 1-8 with median processes. I. Right thoracic endopod 3, rostral. J-M. Details of endopod 3 (I) showing modified spines (J-K) of carpus, barbed seta (L) of merus, and whip seta (M) from ischium. $\mathbf{N}-\mathbf{S}$. Series of dactyli with nail in thoracic endopods 1-5, 8; setae omitted. $\mathbf{T}-\mathbf{U}$. Modified spines from dorsolateral infoldings $(\mathrm{T})$ and lateralia $(\mathrm{U})$ of foregut. 
(cornea not included). In lateral view (Fig. 12B) the cornea appears roughly trapezoid with well-rounded edges, length 1.4-1.5 times maximum width.

Antennulae (Fig. 12C-E). Trunk extends $56-62 \%$ its length beyond eyes, $12-14 \%$ beyond antennal scale. Measured without apophysis along dorsal midline, the basal segment is $47-48 \%$ trunk length, median segment $10-18 \%$, and terminal segment $34-43 \%$. Basal segment on basal half of its outer face with two small, stout, barbed setae as in Fig. 2D. Dorsal apophysis with three barbed setae and one whip seta (Fig. 12E). Lateral apophysis with two barbed setae and one smooth seta. Median segment dorsally with large apophysis bearing three barbed setae and one whip seta. Terminal segment 1.3-1.4 times as long as wide. Its mid-dorsal apophysis with four small, barbed setae. Outer antennular flagellum is thicker than inner one by factor of 1.5-1.7 when measured near basis of flagella. Male lobe setose, inserts ventrally close to terminal margin of antennular trunk, length is $22-31 \%$ width of terminal segment of trunk, its width $22-25 \%$.

Antennae (Fig. 12A). Three-segmented antennal peduncle with basal segment $20 \%$ length of peduncle, second segment $47-48 \%$, and third segment $33 \%$. Sympod with tongue-like, terminally broad process on ventral face (as in Fig. 10E).

MouthParts (Fig. 12F-G). Mandibular palp (Fig. 12F-G) three-segmented. Proximal segment without setae, $10-15 \%$ length of palp. Length of median segment 1.6-2.3 times its maximum width and $61-68 \%$ length of palp. Inner margin of median segment with three sparsely barbed setae in subapical to subbasal position, and with 4-6 short setae on proximal third. Basal half of these setae thickened; with unilateral series of stiff barbs; distal half smooth (Fig. 12G). Outer margin of median segment all along with setae; most setae barbed on basal third. No scales present on median segment. Terminal segment strongly setose (most setae below drawing plane in Fig. 12F), this segment 27-28\% palp length. Pars molaris with well-developed grinding surface in both mandibles. Pars incisiva with four teeth, digitus mobilis with three teeth, and pars centralis with 4-5 spiny teeth. Labium as described above for $H$. smithsoniana sp. nov. Maxillula and maxilla as normal in this genus. Outer margin of exopod of maxilla all along with 16 plumose setae, two apical setae larger than remaining ones.

ThORACIC STERnites (Fig. 12H) with short median processes, in sternites $1-3,8$ terminally rounded, in sternites 4-7 ending in small triangular denticles.

Thoracopods (general; Figs 12I-S, 13A). Length of flagella as well as of basal plates increase from exopod 1 to 5-6, and decrease from exopod 6-8. Basal plates (Fig. 13A) expanded, length 1.7-2.1 times width. Outer edge of plates angular, tip narrowly rounded in exopods $1-2$, and pointed in remaining exopods. The first thoracopods with large, leaf-like, smooth epipod. Plumose seta on intersegmental joint between sympod of thoracopod 2 and the corresponding sternite. Basis of endopods 4-8 with a small, lappet-like apophysis on rostral face below endopod (Fig. 13A). Total length increases in endopods 1-6 and decreases in 6-8. Ischium becomes longer and more slender from endopods 1 to 4 , while both features remain subequal among endopods 4-8. Ischium shorter than merus in endopods 1-3 (Fig. 12I) but longer than merus in endopods 4-8 (Fig. 13A). Thoracic endopods with claw 3 longest; claws 1, 4-8 about half length of claw 3; claw 2 miniaturized. Claws 1-4 smooth (Fig. 12N-Q), claws 5-8 subapically unilaterally serrated (Fig. 12R-S). Claws 1, 4 straight (Fig. 12N, Q), claws 2-3 weakly bent (Fig. 12O-P), claws 5-8 strongly bent (Fig. 12R-S). Penes with one barbed plus one smooth seta in about central position (Fig. 13A).

MAXILLIPEDS. First maxilliped as described above for H. smithsoniana sp. nov. Basis of second maxilliped with large, distinctly medially projecting endite. Combined praeischium plus ischium 0.9 times length of merus, carpopropodus plus dactylus 1.1 times merus. Dactylus very large, with dense brush formed 


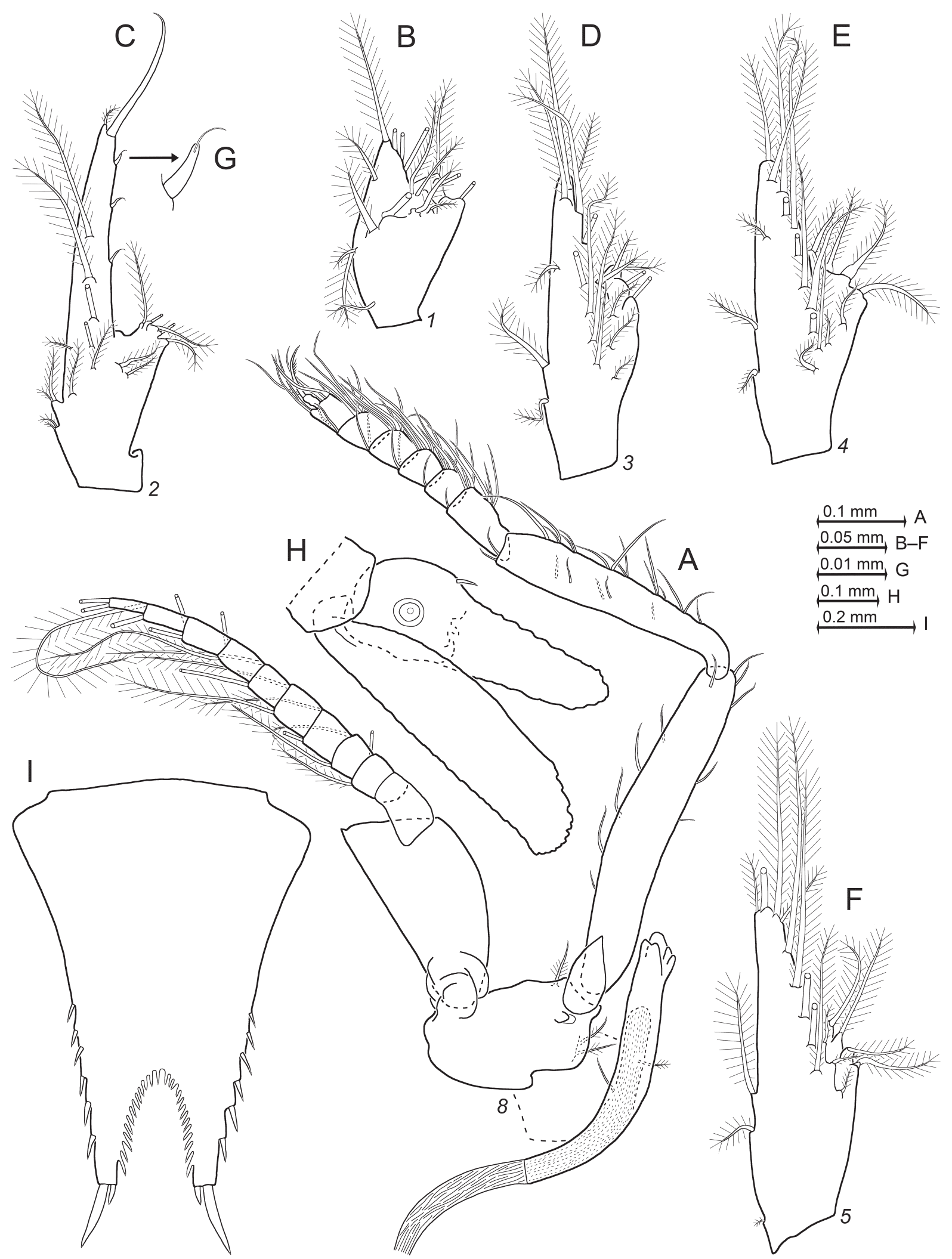

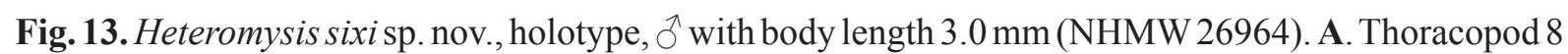
with penis, caudal. B-F. Series of male pleopods 1-5, caudal. G. Detail of male pleopod 2 (C) showing flagellate spine. H. Uropods, ventral; with setae and ventral wall of statocyst omitted. I. Telson. 
by large numbers of normal setae and about ten modified setae, the latter apically bent, bearing two symmetrical series of denticles (stiff barbs) on each side in subbasal to median portions.

Gnathopods (thoracic endopods 3; Fig. 12I-M). Ischium 1.7-2.0 times as long as wide; merus 2.8-3.3 as long as wide and 1.7-2.3 length of ischium. Carpus 0.6-0.7 times length of merus, 0.9-1.1 times ischium. Claw 2.6-3.6 times length of dactylus, and 44-52\% carpopropodus. Distal half of ischium with 3-4 small whip setae (Fig. 12M) on inner margin; these setae inserted on short, terminally rounded projections. Six to eight such setae intermixed in series of larger smooth setae along $60-80 \%$ of inner margin of merus, these setae not inserted on projections. Series of five unilaterally barbed setae (Fig. 12L) near outer margin on distal $3 / 4$ of merus. These setae with series of normal barbs (cilia) along median to subapical portions, basal portions thickened. Carpus with proximal two spines unpaired, distal spines arranged in two pairs; only distal $2-4$ spines with rugged anterior margins.

Pleopods (Fig. 13B-G). Length increases in series of pleopods $1,3 \approx 4,5,2$. For presence and numbers of spines on pleopod 2, see 'Diagnosis' above. All setae of pleopods 1-5 plumose or barbed.

TAIL FAN (Fig. 13H-I). Exopod of uropods reaches with 14-19\% its length beyond endopod and 46-49\% beyond telson. Endopod reaches with $23-26 \%$ its length beyond telson. Statoliths mineralised with fluorite. Statoliths discoidal, diameter 40-42 $\mu \mathrm{m}(\mathrm{n}=2)$. Telson length 1.4 times maximum width, 0.9 times endopod of uropod, 0.7 times exopod of uropod, and 1.3 times last abdominal somite. For further details of telson, see 'Diagnosis' above.

Foregut (Fig. 12T-U) essentially as described above for $H$. smithsoniana sp. nov. Dorsolateral infoldings with two comparatively large, centrally serrated spines (Fig. 12T); posterior part of lateralia with 2-3 smaller, centrally serrated spines (Fig. 12U). The examined gut was almost empty.

\section{Distribution}

The species is so far known only from a service tank of the 'Waikiki Aquarium', Honolulu, Hawaii. Origin most likely in coastal marine waters of the Central Pacific (Table 1).

\section{Discussion}

\section{Validity of Heteromysis (Olivemysis) smithsoniana sp. nov.}

Among species of Heteromysis known to have a modified male pleopod 2, this species shows the greatest number (25-30) of flagellate spines on male pleopod 3. Only three species of Heteromysis previously published in this respect share modified male pleopods $1-4$ with the new species:

Heteromysis (Olivemysis) gomezi Băcescu, 1970, with only males known from two stations in the Caribbean, namely from a sponge in the Batabano Gulf at the southern coast of Cuba (Băcescu 1970) and from corals at the Saba Bank (Brattegard 1980), differs from the new species by a shorter, more broadly rounded rostrum, by an unsegmented carpopropodus of thoracic endopod 3 with fewer flagellate spines ( 3 stout spines and, depending on the interpretation of Băcescu's fig. 1b, an additional 2 slender ones), by carpopropodus 4 with more (5) segments, carpopropodus 7-8 with fewer (5) segments, and by male pleopods $3-4$ with fewer spines (15 and 17-18, respectively).

Heteromysis (Olivemysis) mayana Brattegard, 1970, from diverse habitats in shallow water of the Caribbean and Gulf of Mexico differs from the new species by an undivided antennal scale, an undivided carpopropodus of thoracic endopod 3, the male pleopod 3 with fewer (13-22) spines, and the telson cleft bearing fewer (14-20) laminae. 
Heteromysis (Olivemysis) mclellandi Price \& Heard, 2011, from sponges in fringing reefs off Turks and Caicos Islands, NW-Atlantic, differs from the new species by the carpopropodus of the thoracic endopod 3 with more (7-9) large flagellate spines, the carpopropodus 8 with fewer (6) segments, all five pairs of pleopods modified by attenuated setae in both sexes, the endopod of the uropods with more (4-5) spines, telson with apical cleft whose margins are shorter (15-20\% telson length) and bear fewer (12-15) laminae all along their length.

The male pleopods are unknown in three species of Heteromysis sharing a flagellate spine (as far as known) at the disto-mesial edge of the antennular trunk and series of laminae along $>70 \%$ of the margins of the telson cleft with $H$. smithsoniana sp. nov.:

Only a single female of Heteromysis bredini Brattegard, 1970 is known from Tobago, Caribbean. It differs from the new species by a stouter carpopropodus of the thoracic endopod 3 with more (10) flagellate spines, the endopod of the uropods with more (5) spines, and the lateral margins of the telson with more (19-20) spines.

Only an adult female and one immature male of Heteromysis (Olivemysis) sexspinosa Murano, 1988 are known from a reef flat near Darwin, Australia. It differs from the new species by an undivided antennal scale, the carpopropodus of the thoracic endopod 3 less than twice as long as broad, each lateral margin of the telson all along with more (18-19) spines.

Only two females of Heteromysis kossmanni Nouvel, 1964 are known from the Red Sea (Kossmann 1880). This poorly known species differs from the new species by the female pleopod 1 (if correctly described) terminally with several spines and many setae, the carpopropodus of the thoracic endopod 3 about twice as long as wide, the endopod of the uropod without a spine, the telson with spines all along the lateral margins.

\section{Validity of Heteromysis (Olivemysis) hornimani sp. nov. and H. (O.) waikikensis sp. nov.}

Females with pleopod 2 modified by attenuated setae or by spines were previously found only in H. mclellandi and H. kushimotensis, respectively, and now also in H. hornimani sp. nov. and H. waikikensis sp. nov. Interestingly, the latter three species are known only from public aquaria.

Only Heteromysis (Olivemysis) kushimotensis Murano \& Fukuoka, 2003, from exhibition tanks in the Aquarium of Kushimoto Marine Park Center, Wakayama, Japan, shares a flagellate spine at the distomesial edge of the antennular trunk and modified male pleopods 2-4 in combination with the modified female pleopod 2 with both new species. It differs from both species by the undivided antennal scale, fewer flagellate spines (one in males, two in females) on the mesial margin of the pleopod 2, and by the modified male pleopod 5. In addition, it differs from H. hornimani sp. nov. by only one spine on the endopod of the uropods and by smooth basal half of the lateral margins of the telson.

Heteromysis (Olivemysis) waikikensis sp. nov. differs from H. (Olivemysis) hornimani sp. nov. by shorter flagellate spines on the pleopod 2 in both sexes, fewer (2-7 vs 8-14) flagellate spines on the male pleopod 3, fewer (11-12 vs 21-28) flagellate spines on the male pleopod 4, by only one spine on the endopod of the uropods, and by a smooth basal half of the lateral margins of the telson.

The modified male pleopods 2-4 of Heteromysis tattersalli Nouvel, 1942, from the Cape Verde Islands (NE Atlantic) were initially overlooked by Nouvel $(1942,1943)$ and later communicated to O.S. Tattersall (1967: 190) without indicating the kind of modification. Differences of this damaged, single male specimen from both new species are the telson cleft with fewer (10-12) laminae. In addition, it differs from $H$. waikikensis sp. nov. by the endopod of the uropods with more (3-4) spines, and from 
H. hornimani sp. nov. by the lateral margins of the telson with fewer (8-9) spines, which are distributed only on the distal half.

\section{Validity of Heteromysis (Olivemysis) sixi sp. nov.}

The only adult male known is nearly unique within its genus by having only the pleopod 2 modified by spines: the remaining pleopods have setae only. This pattern was previously found only in small adult males of $H$. (O.) dardani, whose pleopods 3-4 are also modified in normal, larger males (Wittmann 2008).

Heteromysis (Olivemysis) dardani Wittmann, 2008 is associated with diogenid hermit crabs on the Island of Madeira (NE Atlantic). It differs from H. sixi sp. nov. by one apically barbed spine (modified seta) at the dorsal apophysis of the basal segment of the antennula, by a subdivided antennal scale, by the absence of a large smooth spine (stylet) on the apex of the male pleopod 2, by pleopods 3-4 modified in most males, by more (3) spines on the endopod of the uropods, and fewer (21-23) laminae in the telson cleft.

Male pleopods are unknown in three species of Heteromysis sharing the flagellate spine (as far as known) at the disto-mesial edge of the antennular trunk and a series of laminae along $>70 \%$ of the margin of the telson cleft with $H$. sixi sp. nov.:

Only a single female of Heteromysis bredini Brattegard, 1970 is known from Tobago, Caribbean. It differs from the new species by the subdivided antennal scale, the stouter carpopropodus of the thoracic endopod 3 with more (10) flagellate spines, the endopod of the uropods with more (5) spines, and the lateral margins of the telson with more (19-20) spines distributed all along the lateral margins.

Only one adult female and one immature male of Heteromysis (Olivemysis) sexspinosa Murano, 1988 are known from a reef flat near Darwin, Australia. It differs from the new species by the carpopropodus of the thoracic endopod 3 less than twice as long as broad, the endopod of the uropods with more (2) spines, the lateral margin of the telson all along with more (18-19) spines.

Only two females of Heteromysis kossmanni Nouvel, 1964 are known from the Red Sea (Kossmann 1880). This poorly known species differs from the new species by the carpopropodus of the thoracic endopod 3 about twice as long as broad, the endopod of the uropod without spine, and the telson with spines all along the lateral margins.

\section{Acknowledgements}

The authors are greatly indebted to Dominique Barthelmy (Brest), Jamie Craggs (London), Anaïs Courtet (Paris), Bill Hoffman (Fort Pierce), Jakub Kordas (Wrozlaw), and Gwen Lentes (Honolulu) for important aquarium data and for the efforts they made with sampling and sending mysid material to Vienna. Sincere thanks to Katie Ahlfeld (Washington, DC) and Bill Hoffman (Fort Pierce) for information about aquarium exhibitions at the Smithsonian National Museum of Natural History. Santiago Gaviria-Melo (Vienna) kindly determined copepods encountered in aquaria.

\section{References}

Ariani A.P., Wittmann K.J. \& Franco E. 1993. A comparative study of static bodies in mysid crustaceans: Evolutionary implications of crystallographic characteristics. Biological Bulletin 185 (3): 393-404. https://doi.org/10.2307/1542480

Băcescu M. 1968. Heteromysini nouveaux des eaux cubaines: Trois espèces nouvelles de Heteromysis et Heteromysoides spongicola n.g. n.sp. Revue roumaine de Biologie - Zoologie 13 (4): 221-237. 
Băcescu M. 1970. New spongicolous Heteromysis of the Caribbean Sea (H. gomezi n.sp. and H. mariani n.sp.). Revue roumaine de Biologie - Zoologie 15 (1): 11-16.

Băcescu M. 1981. Problèmes de systématique évolutive concernant quelques Crustacés de la Mer Noire. Journées d'Études sur la Systématique évolutive et la Biogéographie en Méditerranée, C.I.E., S.M. 1980: 85-88.

Băcescu M. \& Iliffe T.M. 1986. Bermudamysis g.n., Platyops g.n. and other mysids from Bermudian caves. Stygologia 2 (1-2): 93-104.

Bowman T.E \& Orsi J.J. 1992. Deltamysis holmquistae, a new genus and species of Mysidacea from the Sacramento-San Joaquin estuary of California (Mysidae: Mysinae: Heteromysini). Proceedings of the Biological Society of Washington 105 (4): 733-742.

Brattegard T. 1969. Marine biological investigations in the Bahamas 10. Mysidacea from shallow water in the Bahamas and southern Florida. Part 1. Sarsia 39 (1): 17-106. https://doi.org/10.1080/00364827.1969.10411155

Brattegard T. 1980. Platymysis facilis gen. et sp. nov. (Crustacea: Mysidacea: Heteromysini) from the Saba Bank, Caribbean Sea. Sarsia 65 (1): 49-52. https://doi.org/10.1080/00364827.1980.10431471

Bravo M.R. \& Murano M. 1996. A new species of the genus Pseudomysidetes (Mysidacea) from Japan and reallocation of this genus to the Heteromysini. Crustaceana 69 (4): 476-484. https://doi.org/10.1163/156854096X01050

Hanamura Y. \& Kase T. 2001. Two new shallow-water mysids of the genus Heteromysis (Crustacea: Mysidacea) from a submarine cave of Christmas Island, Eastern Indian Ocean. Species Diversity 6: $11-21$.

Klepal W. \& Kastner R.T. 1980. Morphology and differentiation of non-sensory cuticular structures in Mysidacea, Cumacea and Tanaidacea (Crustacea, Peracarida). Zoologica Scripta 9 (4): 271-281. https://doi.org/10.1111/j.1463-6409.1980.tb00667.x

Kobusch W. 1998. The foregut of the Mysida (Crustacea, Peracarida) and its phylogenetic relevance. Philosophical Transactions of the Royal Society. B. Biological Sciences 353: 559-581. https://doi.org/10.1098/rstb.1998.0227

Kossmann R. 1880. III. Malacostraca (2. Theil Anomura). In: Königliche Academie der Wissenschaften zu Berlin (ed.), Zoologische Ergebnisse einer im Auftrage der königlichen Academie der Wissenschaften zu Berlin ausgeführten Reise in die Küstengebiete des Rothen Meeres 2 (1): 66-140. Wilhelm Engelmann, Leipzig.

Modlin R.F. 1987. Heteromysini from Grand Bahama Island: Description of Heteromysis agelas, new species, first description of male H. floridensis, and notes on H. guitarti (Crustacea: Mysidacea). Proceedings of the Biological Society of Washington 100 (2): 296-301.

Murano M. 1988. Heteromysids (Crustacea; Mysidacea) from northern Australia with description of six new species. The Beagle, Records of the Northern Territory Museum of Arts and Sciences 5 (1): 27-50.

Murano M. \& Fukuoka K. 2003. Two new species of the genus Heteromysis (Crustacea: Mysida: Mysidae) occurred in the aquarium of the Kushimoto Marine Park Center, Japan. Bulletin of the National Science Museum. Series A: Zoology 29 (4): 185-196.

Nouvel H. 1942. Diagnoses préliminaires de Mysidacés nouveaux provenant des campagnes du Prince Albert 1er de Monaco. Bulletin de l'Institut océanographique de Monaco 831: 1-12.

Nouvel H. 1943. Mysidacés provenant des campagnes du Prince-Albert $\mathrm{I}^{\mathrm{er}} \mathrm{de}$ Monaco. In: Richard J. (ed.) Résultats des campagnes scientifiques accomplies sur son yacht par Albert I'r. Fasc. 105: 1-128. Imprimerie de Monaco. 
Pillai N.K. 1968. Heteromysis zeylanica Tattersall (Crustacea: Mysidacea), an associate of Madreporarian corals in South Indian waters. Journal of the Bombay Natural History Society 65 (1): 45-57.

Price W.W. \& Heard R.W. 2011. Two new species of Heteromysis (Olivemysis) (Mysida, Mysidae, Heteromysinae) from the tropical northwest Atlantic with diagnostics on the subgenus Olivemysis Băcescu, 1968. Zootaxa 2823: 32-46. https://doi.org/10.11646/zootaxa.2823.1.2

San Vicente C. \& Monniot F. 2014. The ascidian-associated mysid Corellamysis eltanina gen.nov., sp.nov. (Mysida, Mysidae, Heteromysinae): a new symbiotic relationship from the Southern Ocean. Zootaxa 3780 (2): 323-346. https://doi.org/10.11646/zootaxa.3780.2.6

Schlacher T.A., Wittmann K.J. \& Ariani A.P. 1992. Comparative morphology and actuopalaeontology of mysid statoliths (Crustacea, Mysidacea). Zoomorphology 112 (2): 67-79. https://doi.org/10.1007/BF01673808

Tattersall O.S. 1962. Report on a collection of Mysidacea from South African off-shore and coastal waters (1957-59) and from Zanzibar (1961). Proceedings of the Zoological Society of London 139 (2): 221-247. https://doi.org/10.1111/j.1469-7998.1962.tb01828.x

Tattersall O.S. 1967. A survey of the genus Heteromysis (Crustacea: Mysidacea) with descriptions of five new species from tropical coastal waters of the Pacific and Indian Ocean, with a key for the identification of the known species of the genus. Transactions of the Zoological Society of London 31: 157-193. https://doi.org/10.1111/j.1096-3642.1967.tb00366.x

Tattersall W.M. \& Tattersall O.S. 1951. The British Mysidacea. Ray Society, Monograph 136: 1-460. The Ray Society, London.

Wilson G.D.F. 1989. A systematic revision of the deep-sea subfamily Lipomerinae of the isopod crustacean family Munnopsidae. Bulletin of the Scripps Institution of Oceanography 27: 1-138.

Wittmann K.J. 1978. Adoption, replacement and identification of young in marine Mysidacea (Crustacea). Journal of Experimental Marine Biology and Ecology 32 (3): 259-274.

https://doi.org/10.1016/0022-0981(78)90120-X

Wittmann K.J. 1981. Comparative biology and morphology of marsupial development in Leptomysis and other Mediterranean Mysidacea (Crustacea). Journal of Experimental Marine Biology and Ecology 52 (2-3): 243-270. https://doi.org/10.1016/0022-0981(81)90040-X

Wittmann K.J. 2000. Heteromysis arianii sp.n., a new benthic mysid (Crustacea, Mysidacea) from coralloid habitats in the Gulf of Naples (Mediterranean Sea). Annalen des Naturhistorischen Museums in Wien 102B: 279-290.

Wittmann K.J. 2008. Two new species of Heteromysini (Mysida, Mysidae) from the Island of Madeira (N.E. Atlantic), with notes on sea anemone and hermit crab commensalisms in the genus Heteromysis S.I. Smith, 1873. Crustaceana 81 (3): 351-374. https://doi.org/10.1163/156854008783564037

Wittmann K.J. 2013. Mysids associated with sea anemones from the tropical Atlantic: descriptions of Ischiomysis new genus, and two new species in this taxon (Mysida: Mysidae: Heteromysinae). Crustaceana 86 (4): 487-506. https://doi.org/10.1163/15685403-00003166

Wittmann K.J. \& Abed-Navandi D. 2019. A new species of Heteromysis (Mysida: Mysidae) from public coral reef aquaria in Vienna, Austria. Crustacean Research 48: 81-97. https://doi.org/10.18353/crustacea.48.0_81

Wittmann K.J. \& Griffiths C.L. 2018. A new species of Mysidopsis G.O. Sars, 1864 from the Atlantic coast of South Africa, with supplementary descriptions of two additional species and notes on colour and feeding apparatus (Mysida: Mysidae). Journal of Crustacean Biology 38 (2): 215-234. https://doi.org/10.1093/jcbiol/rux118 
Wittmann K.J. \& Wirtz P. 2017. Heteromysis sabelliphila sp. nov. (Mysida: Mysidae: Heteromysinae) in facultative association with sabellids from the Cape Verde Islands (subtropical N.E. Atlantic). Crustaceana 90 (2): 131-151. https://doi.org/10.1163/15685403-00003624

Wittmann K.J., Schlacher T.A. \& Ariani A.P. 1993. Structure of Recent and fossil mysid statoliths (Crustacea, Mysidacea). Journal of Morphology 215: 31-49. https://doi.org/10.1002/jmor.1052150103

Manuscript received: 20 October 2020

Manuscript accepted: 8 December 2020

Published on: 23 February 2021

Topic editor: Rudy C.A.M. Jocqué

Desk editor: Kristiaan Hoedemakers

Printed versions of all papers are also deposited in the libraries of the institutes that are members of the EJT consortium: Muséum national d'histoire naturelle, Paris, France; Meise Botanic Garden, Belgium; Royal Museum for Central Africa, Tervuren, Belgium; Royal Belgian Institute of Natural Sciences, Brussels, Belgium; Natural History Museum of Denmark, Copenhagen, Denmark; Naturalis Biodiversity Center, Leiden, the Netherlands; Museo Nacional de Ciencias Naturales-CSIC, Madrid, Spain; Real Jardín Botánico de Madrid CSIC, Spain; Zoological Research Museum Alexander Koenig, Bonn, Germany; National Museum, Prague, Czech Republic.

Supplementary file: Video clip in, recorded by D.A.N., shows a typical fish-free habitat of a population of Heteromysis abednavandii in the Haus des Meeres Aquarium in Vienna, Austria.

https://doi.org/10.5852/ejt.2021.735.1247.3651 